\title{
Progress on Integrated Neutron Diagnostics for Deuterium Plasma Experiments and Energetic Particle Confinement Studies in the Large Helical Device During the Campaigns from FY2017 to FY2019*)
}

\author{
Kunihiro OGAWA ${ }^{1,2)}$, Mitsutaka ISOBE ${ }^{1,2)}$ and Masaki OSAKABE ${ }^{1,2)}$ \\ ${ }^{1)}$ National Institute for Fusion Science, National Institutes of Natural Sciences, Toki 509-5292, Japan \\ ${ }^{2)}$ The Graduate University for Advanced Studies, SOKENDAI, Toki 509-5292, Japan
}

(Received 11 November 2020 / Accepted 9 January 2021)

\begin{abstract}
To achieve a steady-state fusion burning plasma, energetic particle confinement studies have been performed in fusion devices. Deuterium plasma experiments in the Large Helical Device starting from March 2017 have expanded the energetic ion confinement study toward a helical-type fusion reactor. To conduct this study, integrated neutron diagnostics, such as a wide dynamic range neutron flux monitor composed of three sets of detectors, a neutron activation system with two irradiation ends, three vertical neutron cameras, four types of scintillating fiber detectors, and a fast time response neutron fluctuation detector, were installed based on the plan and were working stably, as designed. Moreover, an energetic particle confinement study was advanced by utilizing integrated neutron diagnostics. Furthermore, the results of the energetic particle confinement study using neutron diagnostics obtained from FY2017 to FY2019 are reviewed in this paper.
\end{abstract}

(C) 2021 The Japan Society of Plasma Science and Nuclear Fusion Research

Keywords: Large Helical Device, neutron diagnostics, energetic ion confinement, deuterium experiment

DOI: $10.1585 /$ pfr.16.1102023

\section{Introduction}

For a steady-state fusion burning plasma, energetic ion confinement has been widely discussed because the plasma is sustained by deuterium-tritium fusion-born energetic alpha particles [1]. Close attention has been paid to studying the confinement of energetic particles, such as beam ions and ion cyclotron tail ions [2]. Approaching a steadystate fusion burning plasma, studies of energetic particle confinement in stellarators and helical systems have been led by the Large Helical Device (LHD) [3, 4]. In hydrogen campaigns, the study was mainly conducted using the charge-exchanged neutral particle analyzer [5-13] and escaping energetic ion diagnostics [14-25]. The deuterium experiment was started in March 2017 to obtain further advances in helical-type plasma performances and to understand plasma physics [26,27]. Additionally, the deuterium experiment aims to demonstrate the capability of energetic particle confinement in helical systems toward a steadystate fusion burning plasma. LHD is equipped with intensive neutral beams (NBs), having relatively high acceleration energy. Because the deuterium-deuterium fusion cross-section increases significantly with the energy of the deuteron in this energy range, in NB-heated LHD plasma experiments, neutrons are mainly created by beam-

author's e-mail: kogawa@nifs.ac.jp

*) This article is based on the invited talk at the 37th JSPF Annual Meeting (2020, Matsuyama, online meeting). thermal reactions resulting from powerful NB injection [28]. Therefore, neutrons carry the information regarding the beam ions confined in the plasma core area. The neutron diagnostics of LHD was planned in the construction stage of LHD [29, 30]. Installation of neutron and gammaray detectors were scheduled for measuring the total neutron emission rate $\left(S_{\mathrm{n}}\right)$ and fuel ion temperature as well as enhancing energetic particle confinement studies. Plans for neutron diagnostics began taking shape subsequently in 2010 [31]. Candidate detectors for measuring $S_{\mathrm{n}}$, neutron emission profile, neutron energy spectrum, triton burnup ratio, and high-energy gamma-ray were proposed for executing energetic ion confinement studies. The wide variety of neutron diagnostics available in LHD is comparable with that installed in large tokamaks, i.e., JET, TFTR, and JT-60U [32]. Since the expected $S_{\mathrm{n}}$ has a similar intensity compared with deuterium operations executed in large tokamaks, the energetic particle confinement study conducted in LHD is expected to be well advanced as was done in large tokamaks. To conduct the deuterium experiments, the calibration of the neutron flux monitor (NFM) [33] and characterization of vertical neutron camera (VNC) [34] were performed supported by investigations using a three-dimensional neutron and gamma-ray transport code [35-40]. Section 2 presents a review of the integrated neutron diagnostics used in LHD and representative results of energetic particle confinement study. Ad- 
ditionally, this study is summarized in Sec. 3 .

\section{Integrated Neutron Diagnostics in LHD}

A comprehensive set of neutron diagnostics has been developed the energetic particle confinement studies and radiation management in LHD. We advanced the development and establishment of comprehensive neutron measurement hardware following the strategy illustrated in Table 1. The five systems, i.e., the ex-vessel NFM, neutron activation system (NAS), VNC, scintillating fiber (Sci-Fi) detector, and neutron fluctuation (NF) detector have been regularly utilized from FY2017 to FY2019. An arrangement of diagnostics with NB injectors is depicted in Fig. 1.
In LHD, negative-ion-source-based NB (N-NB) injectors, whose acceleration energy reaches $180 \mathrm{keV}$, tangentially inject and mainly create co- or counter-going transit beam ions, while positive-ion-source-based NB (P-NB) injectors, whose acceleration energy is up to $60 / 80 \mathrm{keV}$, perpendicularly inject and mainly create helically trapped beam ions [41]. Using these intense NB injectors, a study of energetic particle confinement is conducted. The specification of each of the diagnostic and representative results obtained from FY2017 to FY2019 is sequentially described.

\subsection{NFM}

LHD became a radiation generator for implementing deuterium plasma experiments, and therefore, strict and

Table 1 List of comprehensive neutron diagnostics in LHD.

\begin{tabular}{|c|c|c|c|c|c|}
\hline System & No. & Detector & Location & Objects & Physics Information \\
\hline \multirow{3}{*}{ Neutron Flux Monitor (NFM) } & 1 & ${ }^{235} \mathrm{U}$ fission chamber, ${ }^{10} \mathrm{~B}$ counter & Top of LHD & \multirow{3}{*}{ Time-resolved total neutron emission rate } & \multirow{3}{*}{$\begin{array}{l}\text { Fusion output and fusion gain } \\
\text { Global confinement of beam ions }\end{array}$} \\
\hline & 2 & ${ }^{235} \mathrm{U}$ fission chamber, ${ }^{3} \mathrm{He}$ counter & 10-O port & & \\
\hline & 3 & ${ }^{235} \mathrm{U}$ fission chamber, ${ }^{3} \mathrm{He}$ counter & 4-O port & & \\
\hline \multirow{2}{*}{ Neutron Activation System (NAS) } & 1 & In, Al, and Si foils & 2.5-L port & \multirow{2}{*}{ Shot-Integrated DD and DT neutron yield } & \multirow{2}{*}{$\begin{array}{l}\text { Global confinement of beam ions } \\
\text { Global confinement of } 1 \mathrm{MeV} \text { tritons }\end{array}$} \\
\hline & 2 & In, Al, and Si foils & 8-O port & & \\
\hline \multirow{3}{*}{ Vertical Neutron Camera (VNC) } & 1 & Stilbene & 2.5-L port/vertical LOS & \multirow{3}{*}{ Time-resolved neutron emission profile } & \multirow{3}{*}{$\begin{array}{l}\text { Deposition profile of beam ions } \\
\text { Radial transport of beam ions }\end{array}$} \\
\hline & 2 & EJ-410(ZnS:Ag + Plastic) & 1.5-L port/diagonal LOS & & \\
\hline & 3 & EJ-410(ZnS:Ag + Plastic) & 1.5-L port/vertical LOS & & \\
\hline \multirow{4}{*}{ Scintillating Fiber (Sci-Fi) detector } & 1 & LANL Sci-Fi detector & $8-0$ port & \multirow{4}{*}{ Time-resolved DT neutron emission rate } & \multirow{4}{*}{ Global confinement of $1 \mathrm{MeV}$ tritons } \\
\hline & 2 & NIFS Sci-Fi detector & 2.5-L port & & \\
\hline & 3 & TOYAMA Sci-Fi detector & 2.5-L port & & \\
\hline & 4 & Large Sci-Fi detector & 2.5-L port & & \\
\hline \multirow{2}{*}{ Neutron Fluctuation (NF) detector } & 1 & EJ-410(ZnS:Ag + Plastic) & 2.5-L port & \multirow{2}{*}{ Neutron Fluctuation } & \multirow{2}{*}{ Global confinement of beam ions } \\
\hline & 2 & EJ-200 (Plastic) & 2.5-L port & & \\
\hline
\end{tabular}

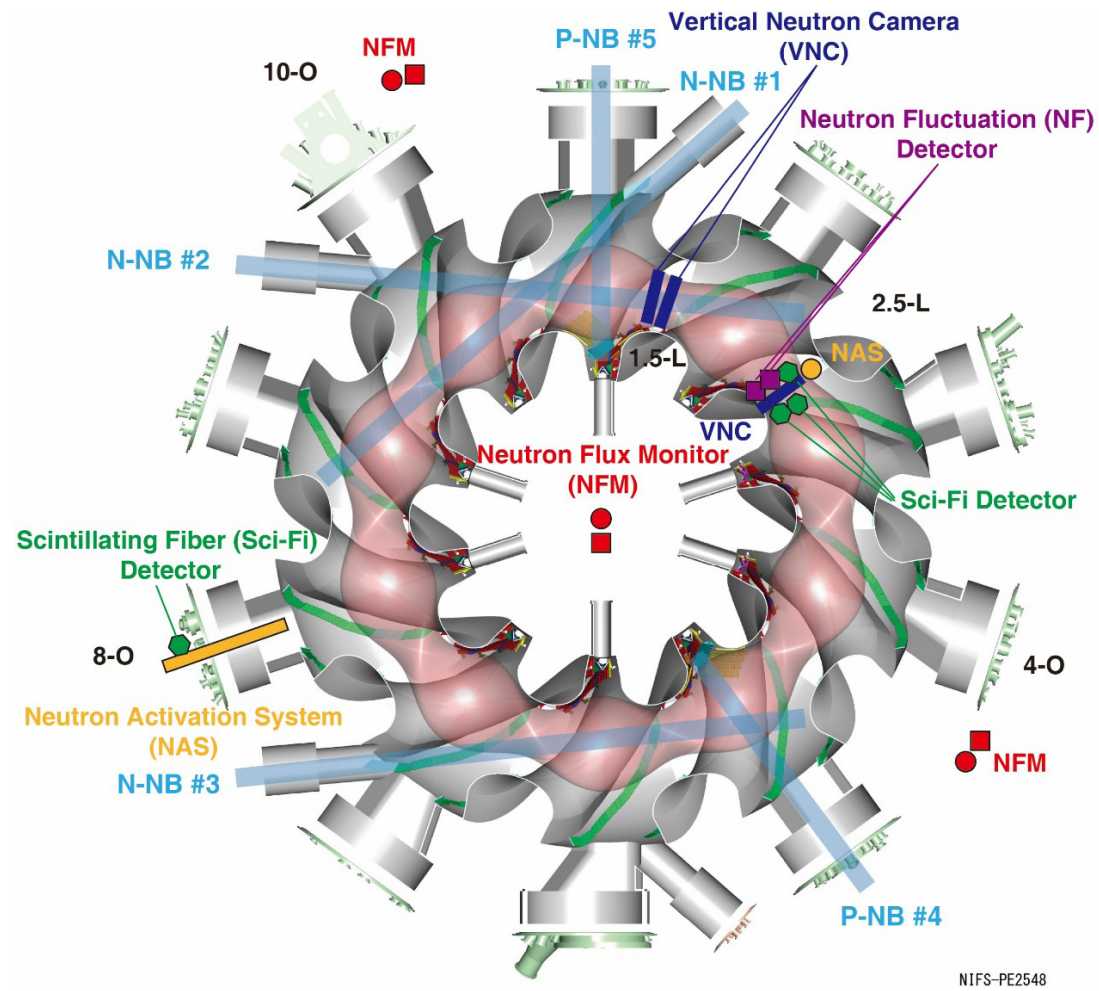

Fig. 1 Arrangement of LHD, NB injectors, and neutron diagnostics. 
stable measurements of weekly, three-month, and annual neutron budgets are necessary. The NFM is classified as the radiation control equipment [42] because the neutron budget in LHD is managed using the NFM. To fulfill the requirements, i.e., wide dynamic range, fast time response, and high noise tolerance for stable measurement of the neutron budget, as well as to perform energetic particle confinement studies, a digital signal processing unit based on innovative field-programmable gate array technology was developed [43,44]. An arrangement of the NFM is shown in Fig. 2 (a) [45]. One set of the detectors is positioned on the top of LHD, while the other two sets are located on the outboard side of LHD. The NFM is comprised of the low $S_{\mathrm{n}}$ range system and the middle to high $S_{\mathrm{n}}$ range system. Further, the low $S_{\mathrm{n}}$ range system is com-

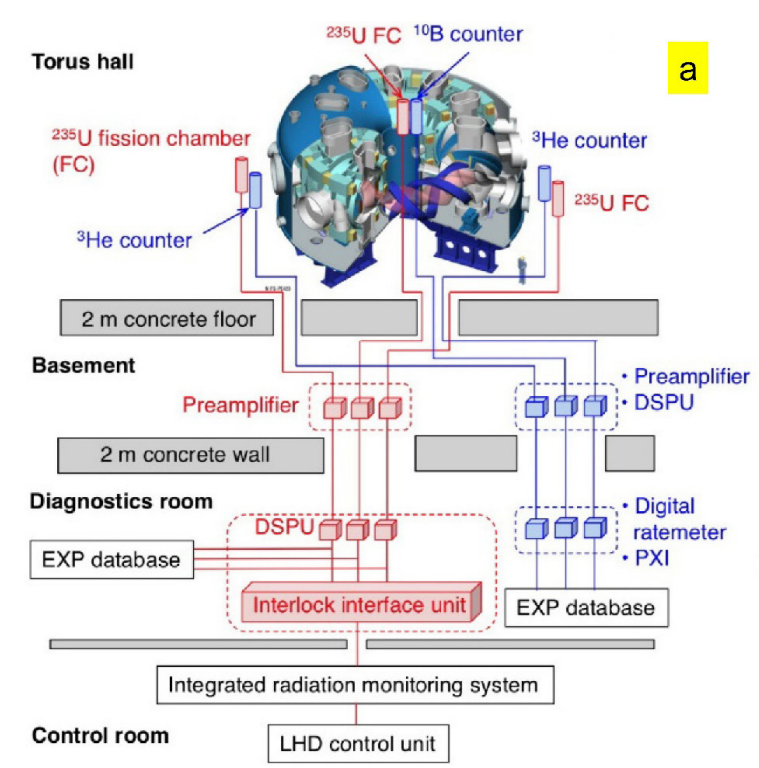

b

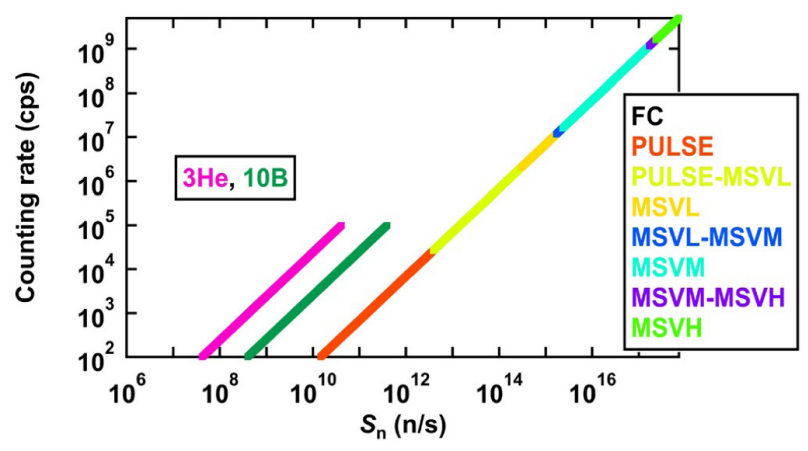

Fig. 2 (a) Schematic diagram of the NFM in LHD. Reproduced with permission from M. Isobe et al., IEEE Trans. Plasma Sci. 46, 2050 (2018) [45]. Here, DSPU represents the digital signal processing unit. (b) Relationship between the counting rate and $S_{\mathrm{n}}$ of each detector of the NFM. Reproduced with permission from M. Isobe et al., IEEE Trans. Plasma Sci. 46, 2050 (2018) [45]. prised of high sensitivity neutron detectors, i.e., one ${ }^{10} \mathrm{~B}$ detector and two ${ }^{3} \mathrm{He}$ detectors are utilized for measuring $S_{\mathrm{n}}$ of up to $5 \times 10^{11} \mathrm{n} / \mathrm{s}$ in order to measure $S_{\mathrm{n}}$ in electron cyclotron heated $(\mathrm{ECH})$ plasmas, for example, while the middle to high $S_{\mathrm{n}}$ range system comprised of ${ }^{253} \mathrm{U}$ fission chambers can measure $S_{\mathrm{n}}$ from $\sim 10^{11} \mathrm{n} / \mathrm{s}$ up to $\sim 5 \times 10^{17} \mathrm{n} / \mathrm{s}$ in order to measure $S_{\mathrm{n}}$ in NB-heated plasmas (Fig. 2 (b)), for example. Note that the ${ }^{235} \mathrm{U}$ fission chamber placed on the top of LHD is regarded as a primary detector to measure $S_{\mathrm{n}}$ as well as manage the neutron amount in LHD. The low $S_{\mathrm{n}}$ range system operates in a pulse counting mode, while the middle to high $S_{\mathrm{n}}$ range system has both pulse counting and Campbelling modes with three ranges in order to achieve a wide dynamic range over six orders of magnitude. In NB-heated deuterium plasma experiments, $S_{\mathrm{n}}$ varies by orders of magnitude within milliseconds based on the NB injection pattern. Additionally, energetic-iondriven magnetohydrodynamic (MHD) instabilities, such as Alfvén eigenmodes [46] and energetic particle modes [47], can lead to energetic ion transport degrading $S_{\mathrm{n}}$ within submillisecond order. Therefore, based on the novel digital signal processing technology, we developed a fast time response ( $0.5 \mathrm{~ms}$ time resolution and $2 \mathrm{~ms}$ time response). For high noise tolerance, we adopted bipolar discrimination in the pulse counting mode for distinguishing negative neutron pulses from bipolar electromagnetic noise, and we newly designed a bandpass filter in the Campbell mode for attenuating electromagnetic noise having frequencies of over $1 \mathrm{MHz}$ [44]. Note that the electrical components of the NFM are located in the basement of the torus hall to avoid the radiation effect on those components [48,49].

The performance of the wide dynamic range NFM was presented in deuterium experiments of LHD. NFM has been stably working even in high $S_{\mathrm{n}}>1 \times 10^{15} \mathrm{n} / \mathrm{s}$ discharges [50]. In addition, the maximum $S_{\mathrm{n}}$ achieved in the first deuterium campaign was $3.3 \times 10^{15} \mathrm{n} / \mathrm{s}$ in the shot number of 140932 (Fig. 3 (a)). In the second deuterium campaign, the shot-integrated neutron yield of $5.0 \times 10^{15}$ was achieved in shot number 144787. In the third deuterium campaign, $S_{\mathrm{n}}$ of $3.2 \times 10^{15} \mathrm{n} / \mathrm{s}$ was achieved in shot number 155422 with a line-averaged electron density of $4.0 \times 10^{19} \mathrm{~m}^{-3}$ by a sudden increase in deuterium density using multiple deuterium pellets. The typical amplitude of an NFM signal is $100 \mathrm{mV}$ order. Therefore, the electromagnetic noise produced by other electrical components could lead to the false detection of pulse counts. Especially, NB injectors operated with high-voltage and highpower can be a strong noise source. To show the robustness against the false detection of pulse counts, we measure $S_{\mathrm{n}}$ in an NB breakdown shot. It was clearly observed that there was no influence on the $S_{\mathrm{n}}$ measurement even if the breakdown of NB occurred in a relatively low-neutron emission discharge, where the NFM is in the pulse counting mode range [44]. Evaluation of the fuel ion temperature was performed in a deuterium plasma without an energetic ion tail, i.e., ECH plasmas using the 

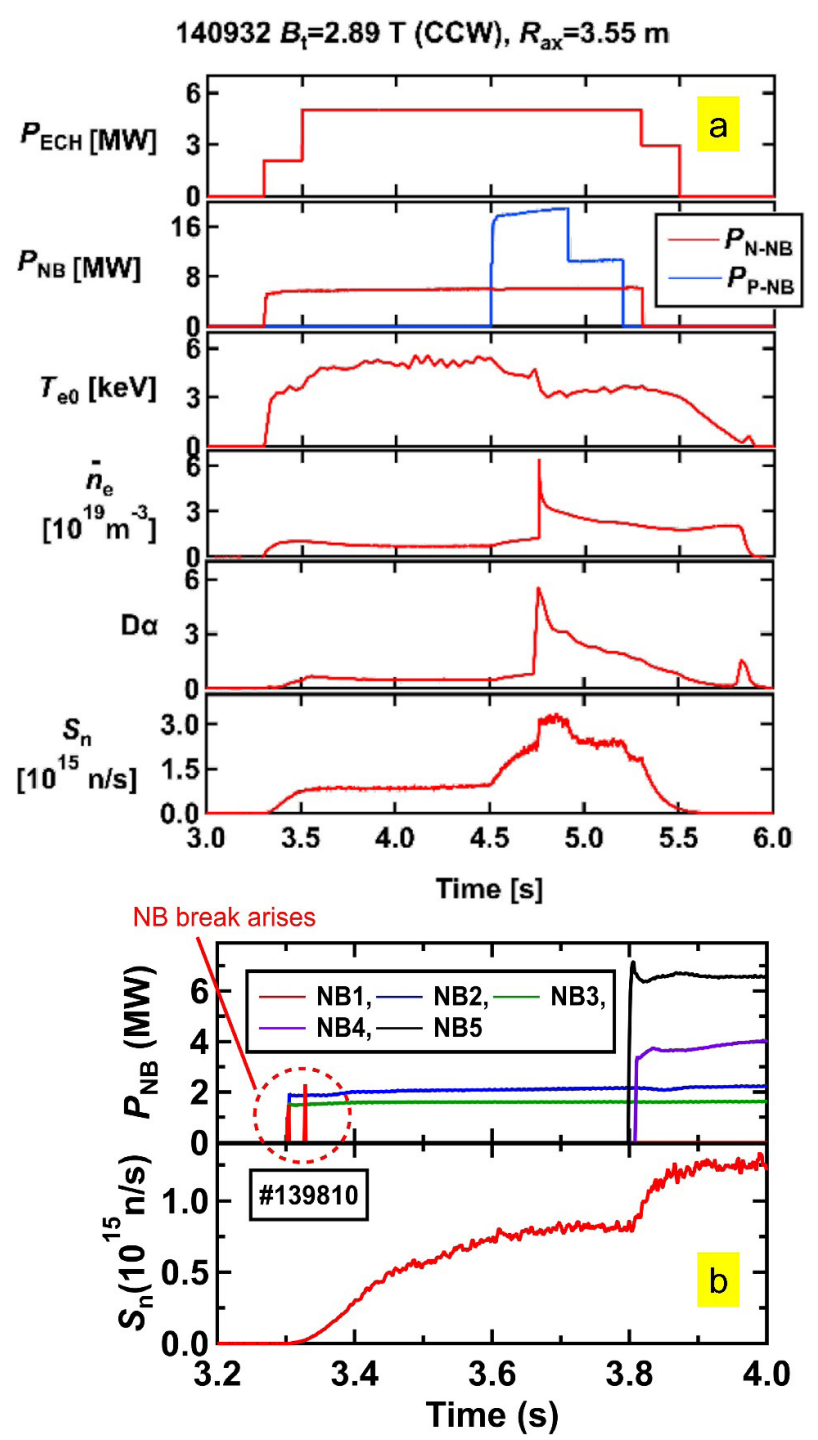

Fig. 3 (a) The discharge in which maximum $S_{\mathrm{n}}$ was obtained in the first deuterium campaign. Reproduced with permission from M. Isobe et al., Nucl. Fusion 58, 082004 (2018) [50]. Here, $P_{\mathrm{ECH}}, P_{\mathrm{NB}}, T_{\mathrm{e} 0}, \bar{n}_{\mathrm{e}}$, and $\mathrm{D} \alpha$ represent injection power of $\mathrm{ECH}$, injection power of $\mathrm{NB}$ injection, the central electron temperature, the line-averaged electron density, and the intensity of $\mathrm{D} \alpha$ emission, respectively. (b) There was no effect on the NFM signal by the NB break down. Reproduced with permission from D. Ito et al., Plasma Fusion Res. 16, 1405018 (2021) [44].

low $S_{\mathrm{n}}$ range system of the NFM, i.e., ${ }^{10} \mathrm{~B}$ detector [51]. In this evaluation, the ion temperature was assumed to have a parabolic radial profile with a Maxwellian distribution. The radial density profile was assumed to have the same shape as the electron density profile. Therefore, $S_{\mathrm{n}}$ can be expressed as a function of the central fuel ion temperature in the calculation. By comparing the experimentally obtained $S_{\mathrm{n}}$ to the $S_{\mathrm{n}}$ calculated based on thermonuclear fusion, we evaluated the central fuel temperature. The central fuel ion temperature evaluated by $S_{\mathrm{n}}$ agrees with the argon-ion temperature measured by ap-

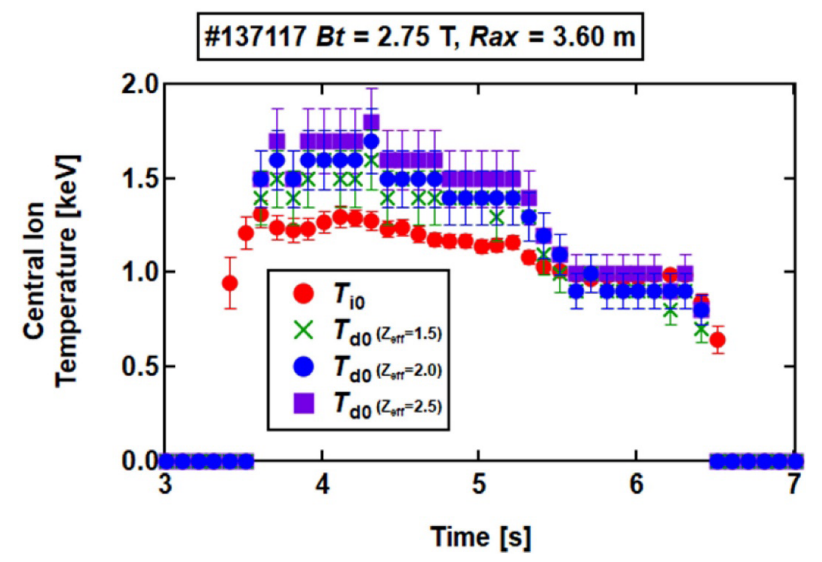

Fig. 4 Time evolution of central fuel ion temperature $\left(T_{\mathrm{d} 0}\right)$ evaluated by total neutron emission rate $\left(S_{\mathrm{n}}\right)$ with different effective charge $\left(Z_{\text {eff }}\right)$ and argon-ion temperature $\left(T_{\mathrm{i} 0}\right)$ evaluated by extreme ultraviolet lithography spectrometer. Reproduced with permission from K. Ogawa et al., Plasma Fusion Res. 12, 1202036 (2017) [51].

plying spectroscopic measurements (Fig.4). To understand the global confinement of energetic ions, using full power NBs and ECH with deuterium gas puff, we surveyed $S_{\mathrm{n}}$ dependence on the plasma density and magnetic axis position $\left(R_{\mathrm{ax}}\right)$. The dependence of the maximum $S_{\mathrm{n}}$ in one discharge on the electron density showed that $S_{\mathrm{n}}$ has a peak around the line-averaged electron density ( $\left.n_{\mathrm{e} \_ \text {avg }}\right)$ from $2 \times 10^{19}$ to $3 \times 10^{19} \mathrm{~m}^{-3}$ (Fig. 5 (a)) [52]. In addition, $S_{\mathrm{n}}$ is found to increase considerably with the inward shift of $R_{\mathrm{ax}}$. Based on the steady-state Fokker-Planck solver FIT3D-DD code [53-55], a comparison between the experimental and numerical simulation results showed that the peak of $S_{\mathrm{n}}$ appeared because of the improvement on NB deposition and the decrease of the beam ion density with the increase of the electron density. A decrease of the beam ion density is caused because of the shorter beam slowingdown time owing to the relatively low electron temperature (Fig. 5 (b)). In addition, the improvement of $S_{\mathrm{n}}$ with an inward shift of $R_{\mathrm{ax}}$ is due to the improvement of the electron temperature at the same density. Furthermore, the effect of resonant magnetic perturbation (RMP) on energetic ion behavior was studied (Fig. 5 (c)) [56]. When we imposed an RMP, $S_{\mathrm{n}}$ decreased regardless of the RMP amplitude in this RMP strength range. With a neutron emission calculation code based on the classical confinement of beam ion FBURN code [57], $S_{\mathrm{n}}$ calculations demonstrated that the degradation of $S_{\mathrm{n}}$ was mainly caused by the degradation of the bulk plasma performance because of RMP (Fig. 5 (d)).

Additionally, using a short pulse NB injection, a study on the classical confinement of beam ions was conducted [58]. Based on the classical confinement of the beam ion, the decay time of $S_{\mathrm{n}}$ after NB stop observed in the experiments was compared with the decay time of $S_{\mathrm{n}}$ evaluated using the CONV_FIT code [59]. The radial diffu- 

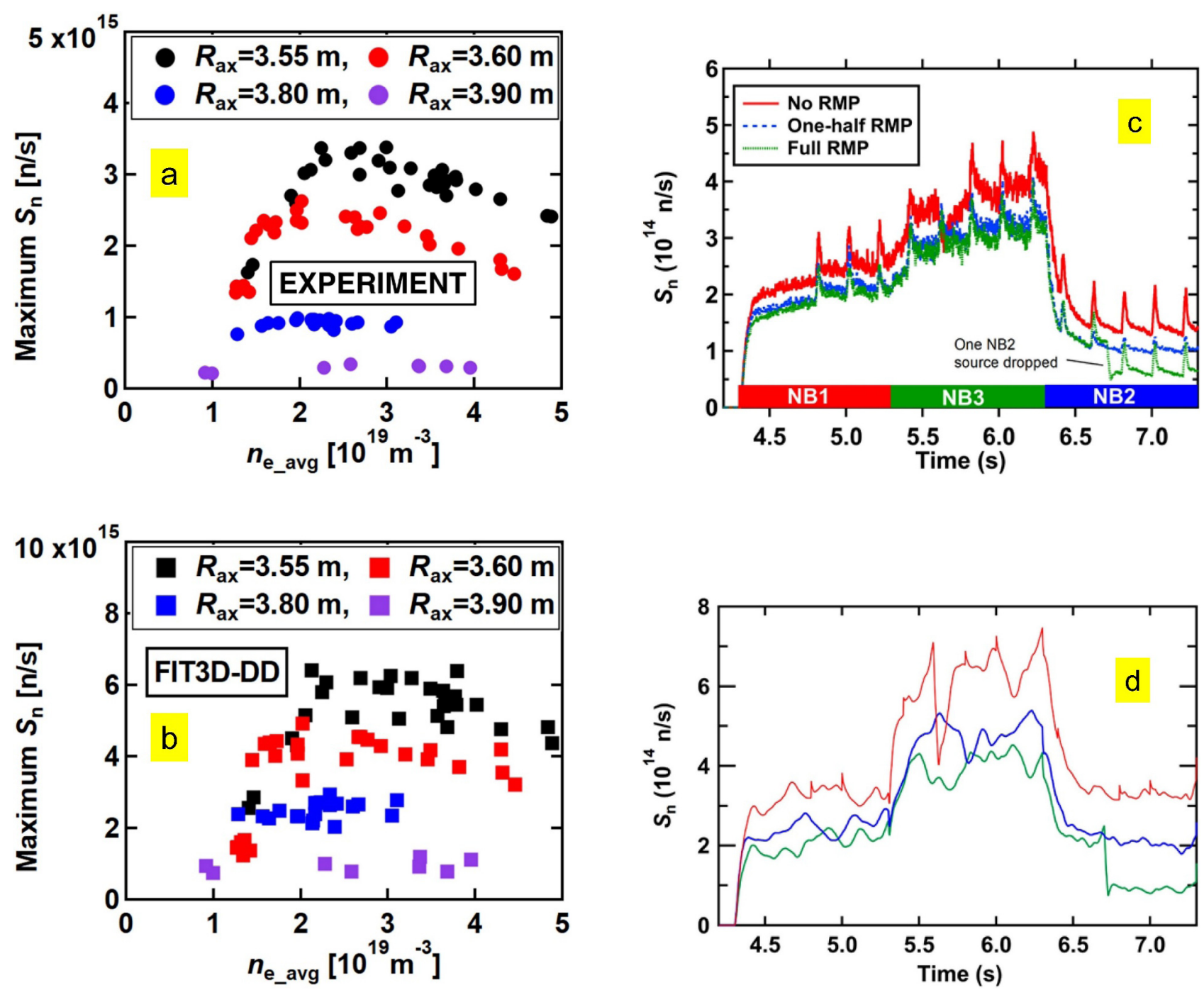

Fig. 5 Dependence of $S_{\mathrm{n}}$ on line-averaged electron density $\left(n_{\mathrm{e} \_ \text {avg }}\right.$ ) (a) obtained from the experiment and (b) calculated using the FIT3DDD code in various plasma axis $\left(R_{\mathrm{ax}}\right)$ conditions. Reproduced with permission from K. Ogawa et al., Nucl. Fusion 59, 076017 (2019) [52]. Time evolution of $S_{\mathrm{n}}$ with no RMP, RMP with one-half amplitude, and full amplitude obtained from (c) experiment and (d) FBURN code. Reproduced with permission from K. Ogawa et al., Plasma Fusion Res. 14, 1202159 (2019) [56].

sion coefficient was estimated to be $0.1-0.3 \mathrm{~m}^{2} / \mathrm{s}$ and 1 $4 \mathrm{~m}^{2} / \mathrm{s}$ for N-NB and P-NB beam ions, respectively [60]. For both N-NB and P-NB ions, the diffusion coefficient becomes smaller with the inward shift of $R_{\mathrm{ax}}$. By considering the effective confinement time and the ratio of the deuteron density to the electron density, the calculated time evolution of $S_{\mathrm{n}}$ agrees with that of the experiment (Fig. 6(a)). Moreover, using the drift kinetic equation solver Global NEoclassical Transport (GNET) code, the time evolution of $S_{\mathrm{n}}$ in short pulse perpendicular P-NB injection was compared in an experiment and the numerical simulation $[61,62]$. The numerical simulation successfully reproduced not only the time trend of $S_{\mathrm{n}}$ but also its absolute value [52]. Thus, we concluded that the behavior of the beam ions injected by a short pulse NB could be described using drift kinetic models in low-beta plasmas without MHD instability. Furthermore, based on the decay time of $S_{\mathrm{n}}$, a study on beam-beam nonlinear Coulomb collision [63] and investigation of knock-on tail formation [64] were also conducted. In fusion plasmas, some kinds of energetic particles, such as fusion born alpha particles, beam ions, and ion cyclotron range of frequency tail ions exist. The interaction between energetic particles may induce the excitation of MHD instabilities through the distortion of the velocity distribution function of energetic particles. However, the conventional Coulomb collision model, which assumes the background Maxwellian, cannot describe the interaction between energetic particles. For this reason, the investigation of this interaction, which can be described by a nonlinear Coulomb collision model, contribute to the understanding of energetic particle confinement. Here, the term "nonlinear collision" comes from the Fokker-Planck equation becomes the nonlinear partial differential equation of the distribution function when the interaction between non-thermal particles is considered $[65,66]$. It was reported that the presence of a hy- 

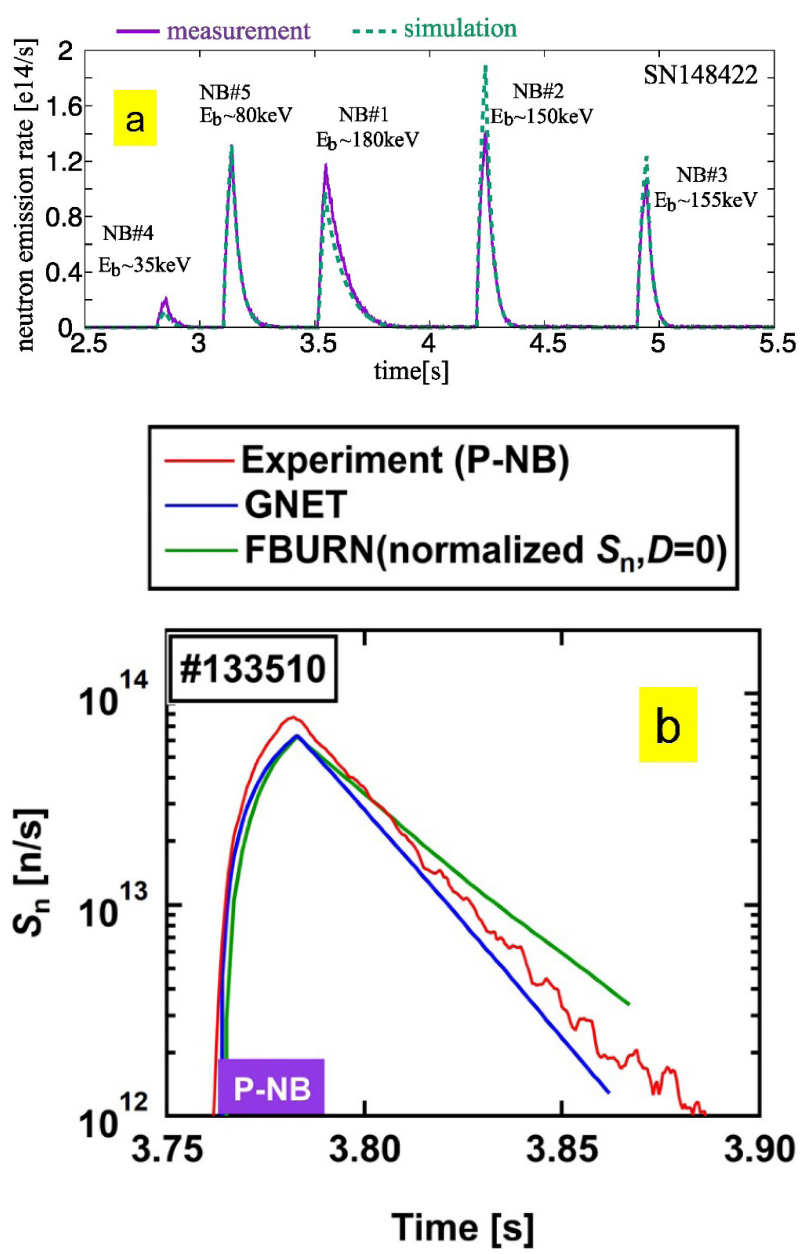

Fig. 6 (a) Time evolution of $S_{\mathrm{n}}$ in the short pulse NB injection obtained from experiment and calculated using the CONV_FIT3D code, including dilution of deuteron and effective confinement time. Reproduced with permission from H. Nuga et al., J. Plasma Phys. 86, 815860306 (2020) [60]. (b) Comparison of the time evolution of $S_{\mathrm{n}}$ in short pulse perpendicular NB injection obtained from the experiment, calculated using the FBURN code, and calculated using the GNET simulation code. Reproduced with permission from K. Ogawa et al., Nucl. Fusion 59, 076017 (2019) [52].

drogen beam, which has the same direction as a deuterium beam, extended the decay time of $S_{\mathrm{n}}$ after the deuterium NB termination compared to the case without the hydrogen beam (Fig. 7 (a)). On the other hand, if the hydrogen beam has an opposite direction to the deuterium beam, almost no effect can be observed the decay time of $S_{\mathrm{n}}$ compared to the no hydrogen beam case. The decay time of $S_{\mathrm{n}}$ calculated using TASK/FP code, where the nonlinear Coulomb collision model is implemented, had a similar trend as the experimental results regarding the hydrogen beam direction. Although $S_{\mathrm{n}}$ and its decay time were overestimated using the TASK/FP code, it was shown that the beam-beam collision effect contributes significantly to $S_{\mathrm{n}}$. The nuclear elastic scattering effect, which is a non-Coulombic scatter-
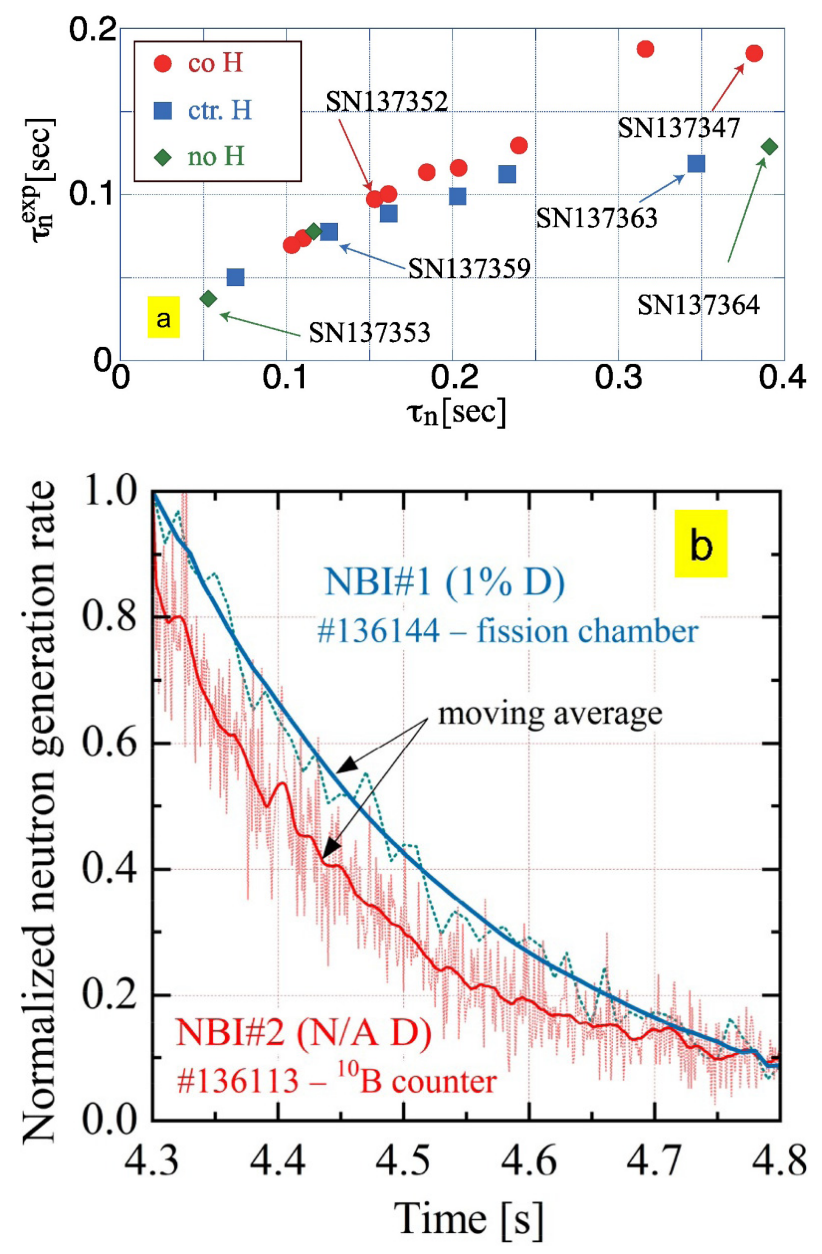

Fig. 7 (a) Dependence of the decay time of $S_{\mathrm{n}}$ after applying deuterium N-NB obtained from an experiment based on the decay time predicted by the TASK/FP code. Moreover, the decay time becomes longer if we inject hydrogen N-NB in the same direction. Reproduced with permission from H. Nuga et al., Nucl. Fusion 59, 016007 (2019) [63]. (b) Time evolution of $S_{\mathrm{n}}$ in N-NB with $1 \%$ deuterium and $0.015 \%$ deuterium (N/A) cases. The decay time of $S_{\mathrm{n}}$ becomes shorter in the $0.015 \%$ deuterium case compared to the $1 \%$ deuterium case. The behavior of the neutron decay times shows that the neutron emission in the $0.015 \%$ case is dominated by the deuteron knockon tail formed by a nuclear elastic scattering effect. Reproduced with permission from $\mathrm{H}$. Matsuura et al., Nucl. Fusion 60, 066007 (2020) [64].

ing process, can introduce an additional heating channel from energetic ions to the bulk plasma. A knock-on tail formed by the nuclear elastic scattering effect due to an N-NB injection was investigated by the deuteron slowing down behavior in the short pulse deuterium P-NB injected plasmas. $S_{\mathrm{n}}$ has a shorter decay time in the case of hydrogen NB injection with approximately $1 \%$ of deuteron compared with that of hydrogen NB injection with natural abundance (N/A) of the deuteron. The decay curve characteristics agree with those predicted by Boltzmann-FokkerPlanck calculation, including the knock-on effect. Such a study is possible owing to the fast time response of NFM. 


\subsection{NAS}

In LHD, access to the torus hall during the operations is strictly limited. We are allowed to enter the LHD torus hall one time each week. If we schedule the special arrangement, c.f., we stop conducting the experiment earlier than usual, we may enter the torus hall at the end of the day based on the dose rate measurement. Therefore, only daily or weekly integrated neutron fluence measurement is possible. Although daily or weekly integrated measurement can contribute to neutron management $[67,68]$, it is obviously insufficient for the study of plasma physics due to the lack of time resolution. Therefore, to measure the neutron fluence shot by shot, we installed the NAS, as planned. Even though the NAS did not give us the time-resolved measurement of neutron flux, the NAS has the advantage of measuring the absolute neutron fluence without gammaray contamination. In particular, in the energetic particle confinement studies, the NAS plays a significant role along with the Sci-Fi detector described in Sec. 2.4 in evaluating the secondary deuterium-tritium DT neutron fluence necessary for the triton burnup study. Further, the system has two irradiation ends composed of the capsule, compressed air tubes, capsule station, pneumatic control system, and two high-purity germanium detectors (Fig. 8 (a)) [69]. An irradiation end was located on the outboard side of LHD (8-O port), while the other irradiation ends were located on the lower side of LHD (2.5-L port). An irradiation end (2.5 L port) was moved to the outboard side of LHD (10-O port) in FY2019 [70]. Moreover, the NAS can carry objects loaded inside the capsule whose inner diameter is $10 \mathrm{~mm}$ and whose weight is less than $10 \mathrm{~g}$.

Using NAS in the early stage of each experimental campaign of LHD, we routinely cross-checked the total neutron yield measurement to confirm the stability of the NFM. By utilizing an indium foil (Fig. 8(b)) in the first deuterium campaign of LHD, the detailed cross-check of the total neutron yield was performed between NFM and NAS [45]. It is indicated that the shot-integrated neutron yield evaluated using NAS linearly increases with the shot integrated neutron yield evaluated by NFM, as intended. In these discharges, neutrons were mainly created by fusion reactions of plasma and beam ions injected by perpendicular P-NB. The linear fitting shows that the slope of the line is 1.08. Therefore, the difference between the NFM and NAS measurements is within $8 \%$. It is well known that the deuterium-deuterium (DD) reaction has directivity. In an experiment using tangential N-NB, directional characteristics of DD neutron emission were predicted to exist using the Fokker-Planck code [71]. Because of the anisotropic effect, the reaction rate of indium can be increased by $25 \%$ and $22 \%$ at the outboard and lower sides of NAS, respectively. Note that for the NFM, the anisotropic effect has been evaluated to be less than $3 \%$. Using the guiding center orbitfollowing models DELTA5D code[72] combined with MCNP6 [73], experiments and detailed analyses were con-
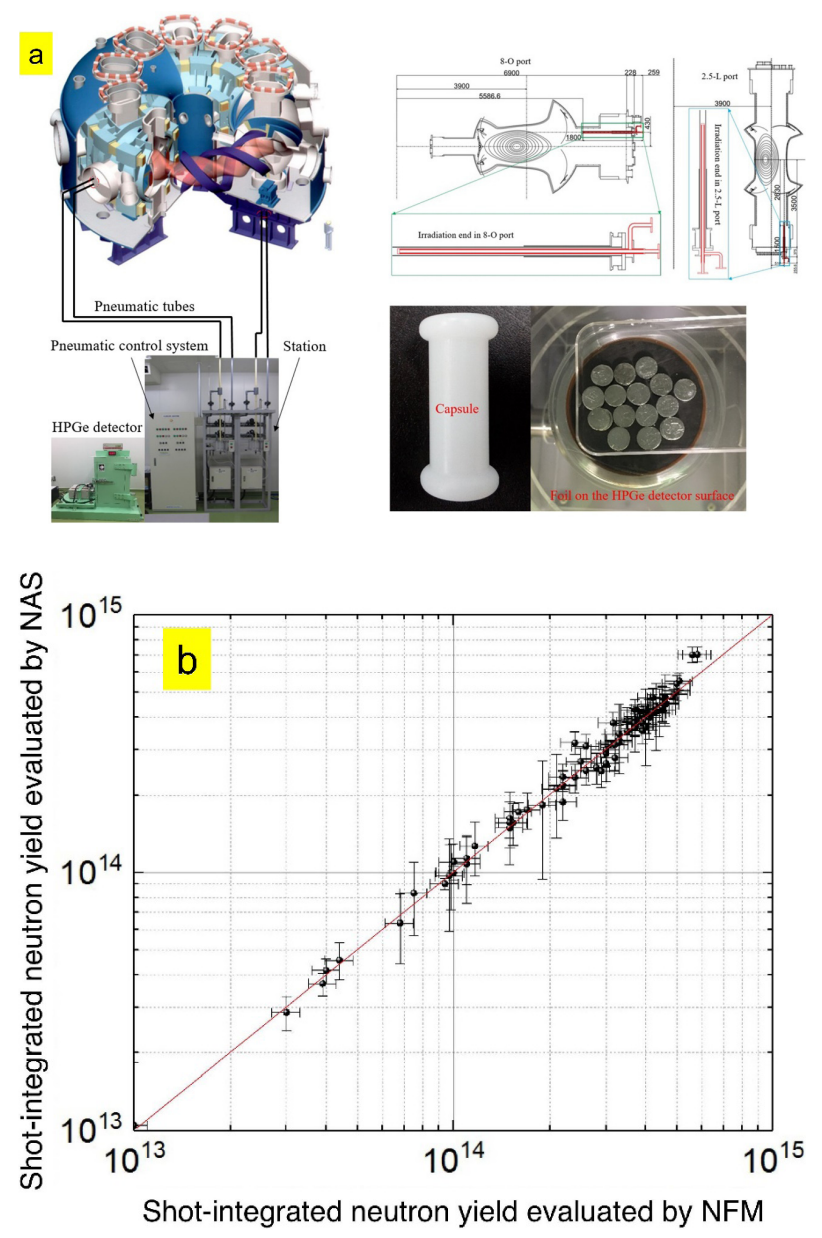

Fig. 8 (a) Schematic diagram of the NAS computer-aided design drawing of irradiation ends. Capsule and indium foil placed on a high-purity germanium detector head. Reproduced with permission from N. Pu et al., Rev. Sci. Instrum. 88, 113302 (2017) [69]. (b) Results of the crosscheck between the NFM and NAS using indium foil. Reproduced with permission from M. Isobe et al., IEEE Trans. Plasma Sci. 46, 2050 (2018) [45].

ducted $[74,75]$. Using indium foil in the different NB injection patterns, i.e., tangential and perpendicular injections, the ratio of neutron fluence was measured using NAS. The ratio of fast-neutron fluence measured at the outboard and lower sides of NAS using the ${ }^{115} \operatorname{In}\left(n, n^{\prime}\right){ }^{115 m}$ In reaction was successfully reproduced by numerical simulations (Fig.9(a)). Using multi foils, such as gold, indium, zinc, aluminum, and silicon with ${ }^{197} \mathrm{Au}(\mathrm{n}, \gamma){ }^{198} \mathrm{Au}$, ${ }^{115} \mathrm{In}(\mathrm{n}, \gamma){ }^{116 \mathrm{~m}} \mathrm{In},{ }^{64} \mathrm{Zn}(\mathrm{n}, \gamma){ }^{65} \mathrm{Zn},{ }^{68} \mathrm{Zn}(\mathrm{n}, \gamma){ }^{69 \mathrm{~m}} \mathrm{Zn},{ }^{115} \mathrm{In}(\mathrm{n}$, n') ${ }^{115 \mathrm{~m}} \mathrm{In},{ }^{64} \mathrm{Zn}(\mathrm{n}, \mathrm{p}){ }^{64} \mathrm{Cu},{ }^{27} \mathrm{Al}(\mathrm{n}, \mathrm{p}){ }^{27} \mathrm{Mg},{ }^{27} \mathrm{Al}(\mathrm{n}, \alpha){ }^{24} \mathrm{Na}$, ${ }^{28} \mathrm{Si}(\mathrm{n}, \mathrm{p}){ }^{28} \mathrm{Al}$, and ${ }^{64} \mathrm{Zn}(\mathrm{n}, 2 \mathrm{n}){ }^{63} \mathrm{Zn}$ reactions, respectively, neutron spectra measurements were performed using the NAS [76] in order to understand the neutron spectra around LHD and to compare the neutron transport calculation. The measured neutron spectra were compared with numerical simulations using the unfolding code SAND-II [77], where the initial guess spectrum was provided by the neu- 

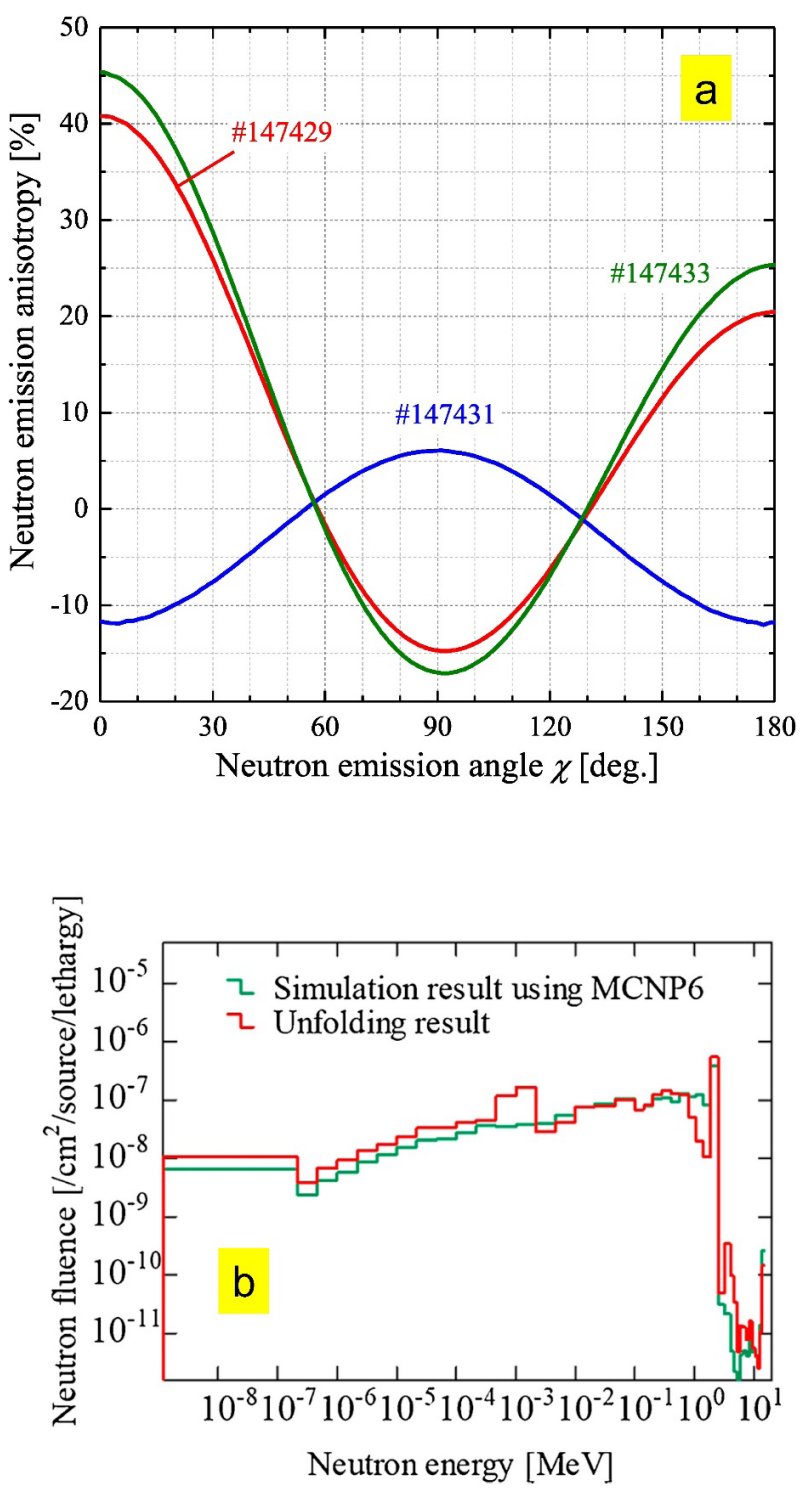

Fig. 9 (a) Neutron emission anisotropy as a function of neutron emission angle relative to the toroidal axis $\chi$ in different NB injection scenarios. Tangential N-NB is injected in shot numbers 147429 and 147433 , while perpendicular P-NB is injected in the shot number 147431. Reproduced with permission from S. Sugiyama et al., Nucl. Fusion 60, 076017 (2020) [75]. (b) Neutron energy spectra expected by MCNP calculation and obtained from the SAND-II code using multiple activation foils measurement results. Reproduced with permission from $\mathrm{T}$. Tanaka et al., Fusion Eng. Des. 146, 496 (2019) [76].

tron transport code MCNP6. It was observed that the calculated neutron energy spectra agree with the experimental results within an order of magnitude range of neutron fluence (Fig. 9(b)).

\section{$2.3 \mathrm{VNC}$}

Visualization of the neutron emission profile was performed in LHD for the first time in a stellarator/helical system. In helical systems, not only co- and counter-passing transit orbits but also helically trapped orbits exist [78]. The helically trapped orbit has a poloidal structure, unlike the passing transit orbit. The confinement of helically trapped ions is always a subject of discussion in energetic particle confinement in helical systems. To visualize the radial profile of the beam ion density in order to understand the confinement of energetic ions as well as transport of energetic ions due to MHD instabilities, VNCs were installed in three locations. A VNC (VNC1) installed in the $2.5-\mathrm{L}$ port was equipped to measure the neutron emission profile in a relatively high-neutron emission rate region, where the expected maximum neutron emission rate is $\sim 10^{16} \mathrm{n} / \mathrm{s}$ (Fig. 10 (a)) [79]. In addition, the others (VNC2 and VNC3) were installed in the 1.5-L port for measuring the neutron emission profile in a relatively low-neutron emission rate range, i.e., $10^{14} \sim 10^{15} \mathrm{n} / \mathrm{s}$, where strong MHD instabilities can be observed [80]. VNC1 has 11 sightlines. Using hematite-doped heavy concrete, having superior radiation shielding performance compared with polyethylene and lead, the multichannel collimator was made inside the hole of the torus hall floor concrete (Fig. 10(b)) [81]. A stilbene scintillator having a pulse shape discrimination ability $[82,83]$, whose diameter and thickness are $20 \mathrm{~mm}$ and $10 \mathrm{~mm}$, respectively, is selected as a fast-neutron scintillator for VNC1. The scintillator is coupled with a photomultiplier tube (H11934100-10MOD, Hamamatsu Photonics KK), characterized by high gain stability in the high pulse counting rate. Further, as shown in Fig. 10 (c), online and offline pulse shape discriminations are simultaneously possible by utilizing the digital signal processor equipped with $1 \mathrm{GHz}$ sampling analog to digital converter and field-programmable gate arrays (APV8102-14MWPSAGb, Techno-AP). VNC2 having nine sightlines was installed in a diagonal poloidal cross-section, while VNC3 having eight sightlines was installed in a vertically elongated poloidal cross-section. Figure 11 describes the sightlines of VNC2 and VNC3 with a typical density profile of helically trapped beam ions calculated by applying the Monte-Carlo orbit following model (MORH) code [84]. Note that the VNC2 is tandemly installed using a chemical vapor deposition single-crystal diamond-based neutral particle analyzer for simultaneous measurement of neutrons and charge-exchanged energetic particles [85]. In VNC2 and VNC3, an EJ-410 scintillator is selected as a fast neutron scintillator having a diameter and thickness of 2 inches and 0.625 inches, respectively. The scintillator is coupled with a conventional photomultiplier tube (H7195, Hamamatsu Photonics K. K.) characterized by high gain stability operated in the current mode. The EJ-410 scintillator volume is almost ten times larger compared with the stilbene scintillator for high-detection efficiency measurement. Besides, the anode signal of the photomultiplier tube $\mathrm{H} 7195$ is fed into the low-impedance current amplifier (C7319, Hamamatsu Photonics K. K.) having a relatively high-frequency response up to $200 \mathrm{kHz}$. By applying an analog to digital converter (PXI-6133, Na- 

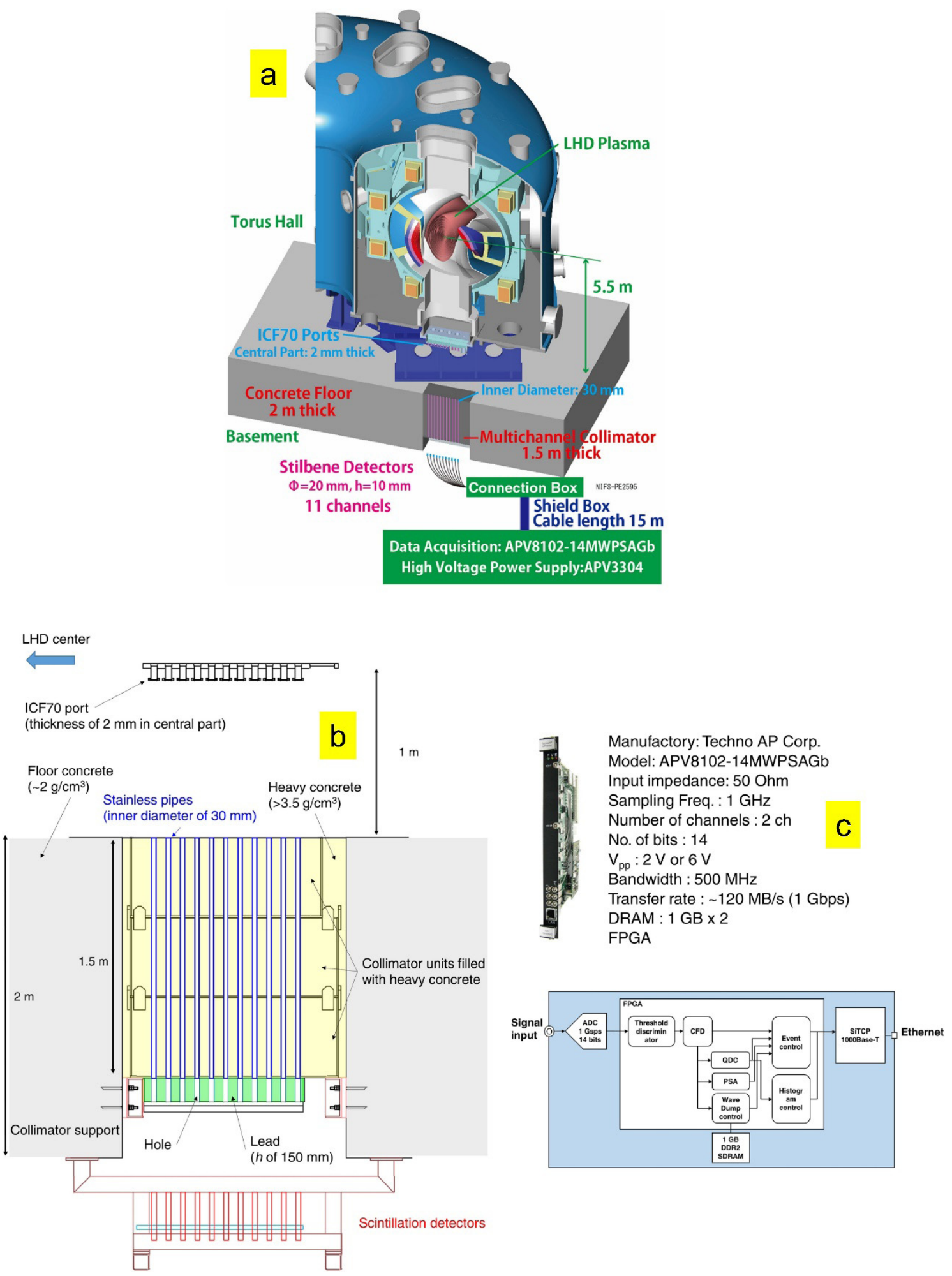

Fig. 10 (a) A schematic diagram of VNC1. Reproduced with permission from K. Ogawa et al., Rev. Sci. Instrum. 89, 113509 (2018) [79]. (b) The detailed structure of multichannel collimator for VNC1. Reproduced with permission from K. Ogawa et al., Rev. Sci. Instrum. 89, 113509 (2018) [79]. (c) Data acquisition system developed for VNC1. Reproduced with permission from K. Ogawa et al., Rev. Sci. Instrum. 89, 113509 (2018) [79].

tional Instruments), the output of the current amplifier is obtained with a sampling frequency of $1 \mathrm{MHz}$.

Measurement of the time-resolved neutron emission profile in N-NB and P-NB-heated plasmas were performed in relatively low-density plasmas using $\mathrm{VNC1}$ [86]. In the experiment, the line-integrated neutron emission profile was measured in MHD quiescent deuterium plasma with deuterium NB heating. In the N-NB-heated plasma case, 

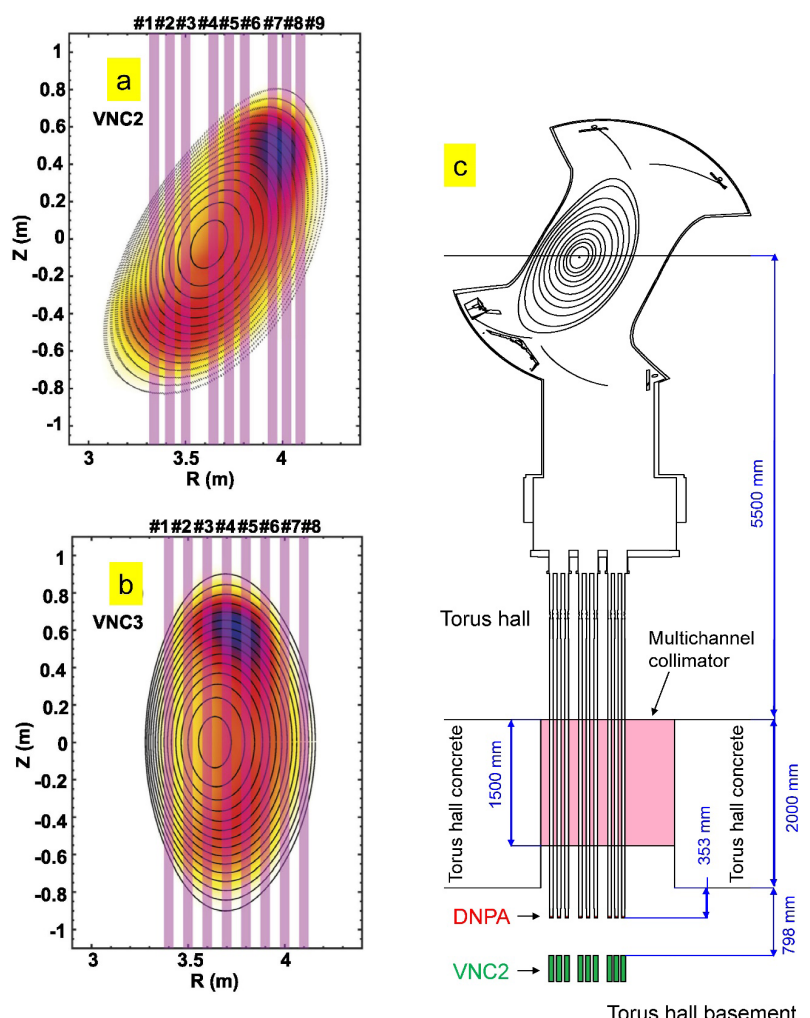

Fig. 11 Sightline of (a) VNC2 and (b) VNC3 along with typical density profile of P-NB ion. Reproduced with permission from S. Sangaroon et al., Rev. Sci. Instrum. 91, 083505 (2020) [80]. Here, P-NB ions orbit was followed until the relaxation time. (c) Arrangement of a diamond neutral particle analyzer (DNPA) array and VNC2.

neutron counts $\left(C_{\mathrm{n}}\right)$ monotonically decrease with $r_{\min } / a$, while in the P-NB-heated plasma case, $C_{\mathrm{n}}$ has a steep gradient at $r_{\text {min }} / a$ of $\sim 0.5$, where $r_{\text {min }}$ and $a$ denote radial distance of a sightline and plasma center and minor radius, respectively (Figs. 12 (a) and (c)) [87]. The measured neutron emission profile is consistent with the beam ion density profile calculated from the MORH code, considering the slowing down of beam ions (Fig. 12 (b), (d)). In high-temperature discharge in LHD, the helically trapped energetic-ion-driven resistive interchange mode (EIC) excited by intensive P-NB injections into relatively low-density plasmas limits the sustainment of the hightemperature state [88-91]. Characteristics of EIC were studied in the deuterium campaign of LHD [92]. The time evolution of $S_{\mathrm{n}}$ shows that its decay time with EIC bursts is significantly shorter than that without EIC burst [93]. Moreover, the drop rate of both $S_{\mathrm{n}}$ and $C_{\mathrm{n}}$ increases with the EIC amplitude. The drop rates of $S_{\mathrm{n}}$ and $C_{\mathrm{n}}$ reach up to $50 \%$ and $60 \%$, respectively with the EIC amplitude of $\sim 0.9$ $\times 10^{-3} \mathrm{~T}$ at the magnetic probe position. The measurement of $\mathrm{VNC} 1$ shows that the EIC decreases neutron counts in the central cords, while the neutron counts in the outer cords are almost unchanged [87]. Therefore, the decrease in neutron counts of central cords indicates the transport
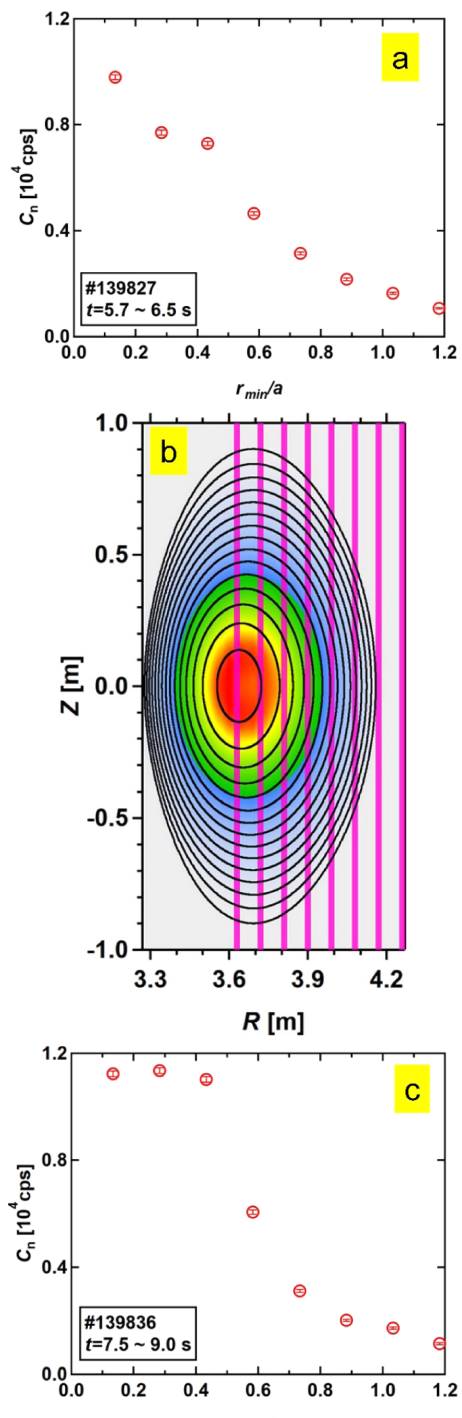

$r_{\text {min }} / a$

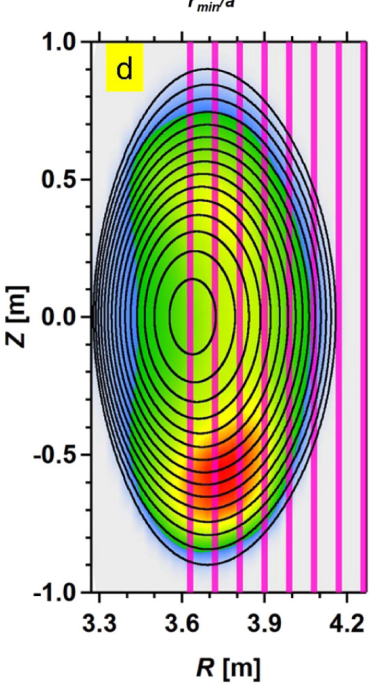

Fig. 12 Line-integrated neutron profile measured by applying VNC1 in (a) N-NB-heated plasma and (c) P-NB-heated plasma. Here $C_{\mathrm{n}}$ indicates neutron pulse counts. The typical beam ion density predicted by utilizing MORH code in (b) N-NB case and (d) P-NB case. Reproduced with permission from K. Ogawa et al., Plasma Phys. Control. Fusion 60, 044005 (2018) [87]. 

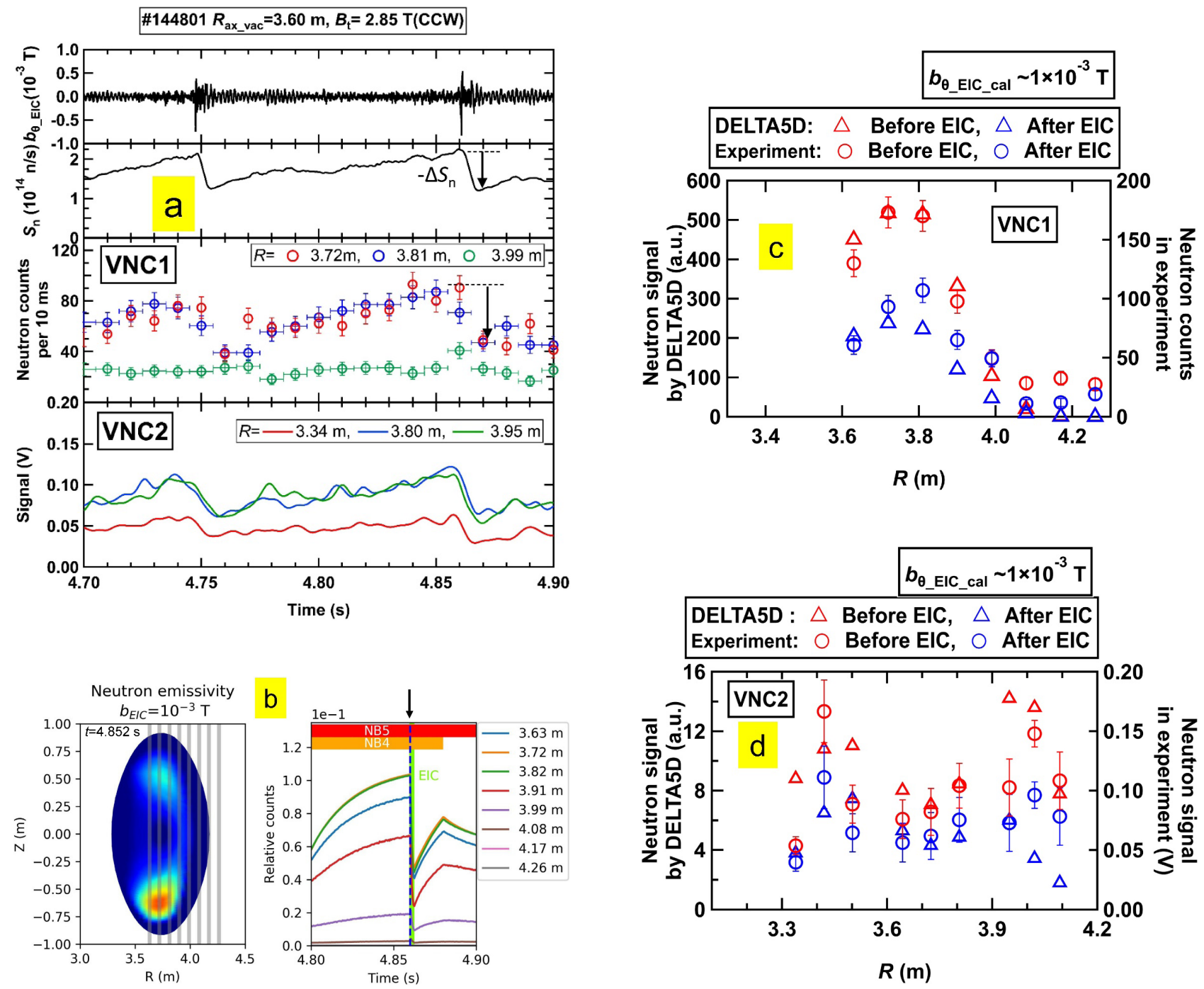

Fig. 13 (a) Time evolution of the EIC experiment. A significant decay of $S_{\mathrm{n}}$ and neutron signals obtained using VNC1 and VNC2 is caused by an EIC burst observed in the magnetic fluctuation amplitude measured by the magnetic probe $\left(b_{\theta_{-} \text {EIC }}\right)$. Reproduced with permission from K. Ogawa et al., Nucl. Fusion 60, 112011 (2020) [94]. (b) Neutron emissivity profile and time evolution of neutron signal calculated by utilizing DELTA5D code. Reproduced with permission from K. Ogawa et al., Nucl. Fusion 60, 112011 (2020) [94]. Line-integrated neutron profile before and after EIC burst measured from experiment and obtained from numerical simulation for (c) VNC1 and (d) VNC2.

of helically trapped beam ions. To understand the helically trapped beam ion transport due to EIC, we conducted additional experiments and numerical simulations in detail using VNC1 and VNC2 [94]. By adding VNC2, the change of the neutron emission profile due to EIC was measured using the fine time resolution (Fig. 13 (a)). Comparison of the orbit-following model using DELTA5D code, including the EIC fluctuation, was performed. Herein, the mode structure of the EIC was given based on electron cyclotron emission measurement [89]. In the conducted experiment, it was shown that the reduction ratio of the signal in $S_{\mathrm{n}}$, signal in central cords of VNC1, and signal in VNC2 cords viewing the helical ripple valley increases with the EIC amplitude (Fig. 13 (b)). The line-integrated neutron emission profile measured by applying VNC1 and VNC2 before and after the EIC burst was compared with the nu- merical simulation. In $\mathrm{VNC} 1$, the neutron counts at the central channels become almost one-half, as observed in the conducted experiment (Fig. 13 (c)). In VNC2, although the neutron profile evaluated in calculation has a relatively wide peak compared with the experiment, the neutron signal corresponding to two helical ripple valleys decreases with the EIC burst, as observed in the conducted experiment (Fig. $13(\mathrm{~d})$ ). Further, it is revealed that helically trapped beam ions up to $60 \%$ immediately escaped from the plasma by EIC excited by helically trapped beam ions.

\subsection{Sci-Fi detector}

In deuterium operations, there is a small fraction of DT neutron emission from plasma deuterium and DD fusion born triton reaction. DD born $1 \mathrm{MeV}$ triton burnup 
experiments have been regarded as the simulation of an alpha particle confinement experiment because kinetic parameters, such as Larmor radius and precession frequency of the $1 \mathrm{MeV}$ triton, are almost the same as $3.5 \mathrm{MeV}$ alpha particles. Based on the Sci-Fi detector used in JT-60U, we developed Sci-Fi detectors for DT neutron measurement in LHD (Fig. 14 (a)) [95-97]. The detector head is made of aluminum substrate embedded with Sci-Fis. We developed three types of Sci-Fi detectors. One so-called NIFS Sci-Fi detector (Fig. 14 (b)) consists of a 1-inch diameter detector head and a 1-inch photomultiplier tube characterized by being relatively strong against magnetic fields (H615270, Hamamatsu Photonics K. K.) [98]. The total number of Sci-Fis is 109 , and the length of each fiber is $10 \mathrm{~cm}$. The second so-called Toyama Sci-Fi detector (Fig. 14 (c)) consists of a $30 \mathrm{~mm} \times 30 \mathrm{~mm}$ detector head and a photomultiplier tube characterized by high gain stability in the high pulse rate (H11934-100MOD, Hamamatsu Photonics K. K.). The total number of Sci-Fis is 144, and the length of each fiber is $6 \mathrm{~cm}$. Further, the length of fibers is chosen based on the experiment and numerical simulation $[99,100]$. The third so-called large Sci-Fi detector (Fig. 14 (d)) consists of a $150 \mathrm{~mm}$ diameter detector head and a 2-inch photomultiplier tube characterized by being relatively strong against magnetic fields (H661470, Hamamatsu Photonics K. K.) [101]. The total number of Sci-Fis is 5156, and the length of each fiber is $5 \mathrm{~cm}$. Additionally, these detectors are developed to operate in relatively low DT neutron rate conditions to study the effect of MHD instability on triton transport and application to fusion machines with a relatively low DT neutron rate [102,103]. The anode signal of Sci-Fi detectors is fed into two types of data acquisition systems. One is a digital signal processor developed for VNC1, while the other is an octal discriminator (APV2500C, Techno AP). We utilized the digital signal processor to determine the discrimination level of DT neutron measurement, and then we mainly used an octal discriminator to obtain the time evolution of the DT neutron emission rate. An octal discriminator can operate at a higher pulse counting rate $(>1 \mathrm{MHz})$ compared with a digital signal processor because of the relatively simple logic and reduced data size of the octal discriminator. Note that the typical pulse width of a Sci-Fi detector is $20 \mathrm{~ns}$. Therefore, the pulse pileup rate in $1 \mathrm{MHz}$ will be $\sim 2 \%$, which is negligibly small.

For the first time, a triton confinement experiment was performed in stellarator/helical devices in LHD [104, 105]. As illustrated in Fig. 15(a), the pulse height spectra of the Sci-Fi detector of NIFS Sci-Fi were obtained. The pulse height spectrum has two components. The lowheight component corresponds to gamma-rays and lowenergy neutrons, while the high-height component corresponds to DT neutron. The result of the time-resolved measurement of the DT neutron emission rate is shown in Fig. 15 (b). Here, the Sci-Fi detector was calibrated by applying a NAS measurement using ten pieces of silicon
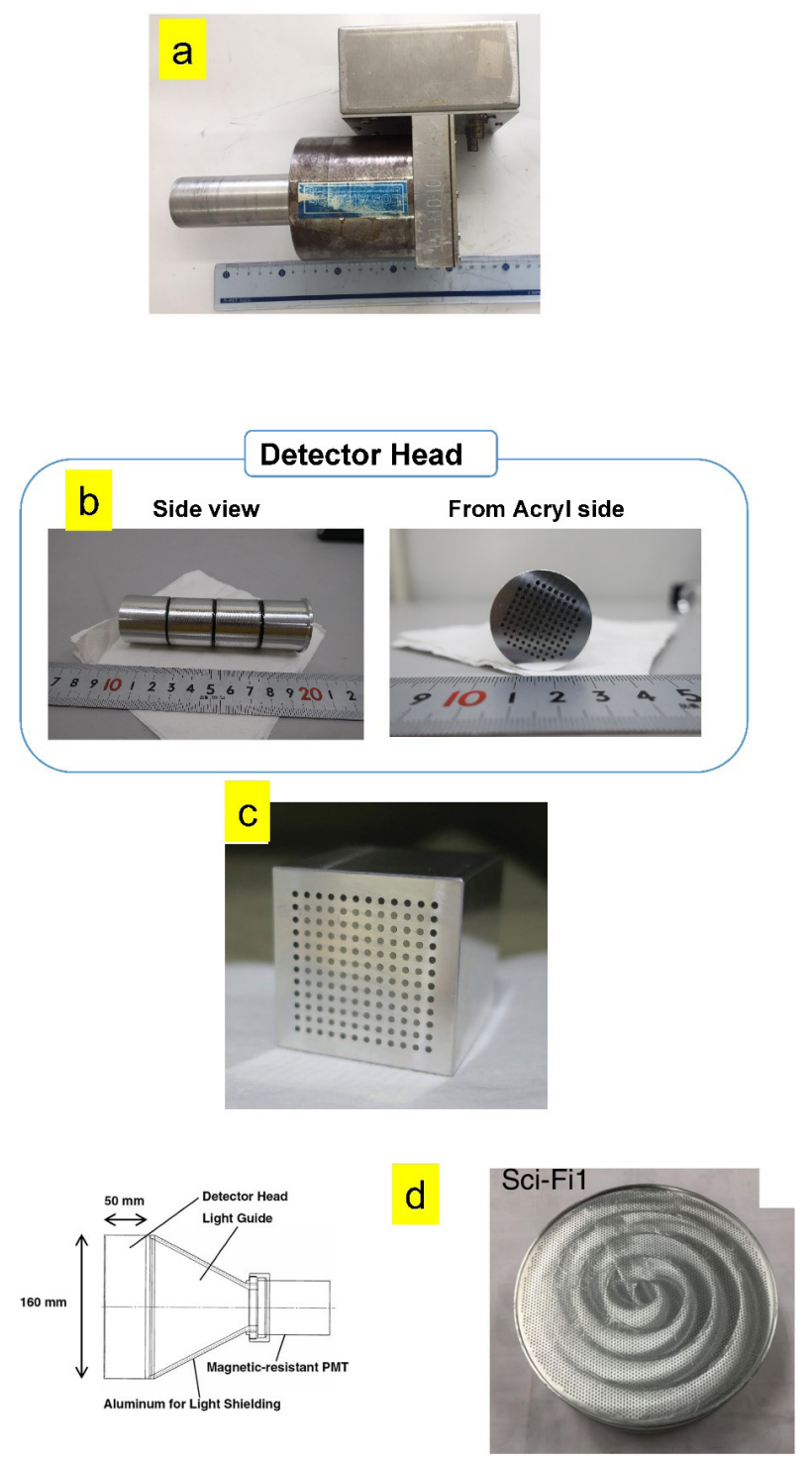

Fig. 14 (a) LANL Sci-Fi detector. Reproduced with permission from N. Pu et al., Rev. Sci. Instrum. 89, $10 \mathrm{I} 105$ (2018) [95]. (b) NIFS Sci-Fi detector. Reproduced with permission from K. Ogawa et al., Nucl. Fusion 58, 034002 (2018) [98]. (c) Toyama Sci-Fi detector. Reproduced with permission from M. Isobe et al., IEEE Trans. Plasma Sci. 46, 2050 (2018) [45]. (d) Large SciFi detector Reproduced with permission from E. Takada et al., Plasma Fusion Res. 11, 2405020 (2016) [99].

foils. Moreover, the DT neutron emission rate rises and decays slowly compared with the DD neutron emission rate. This is in agreement with the characteristics of DD and DT reaction curves. The DD fusion cross-section monotonically increases with the increase of the deuteron energy in this deuteron energy range. Thus, the DD neutron emission rate has a peak at the injection energy for N-NB and P-NB. On the other hand, the DT fusion cross-section has a peak around an energy of $\sim 100 \mathrm{keV}$. Therefore, a $1 \mathrm{MeV}$ triton should be slow downed inside the plasma in order to encounter a DT neutron emission cross-section peak. 

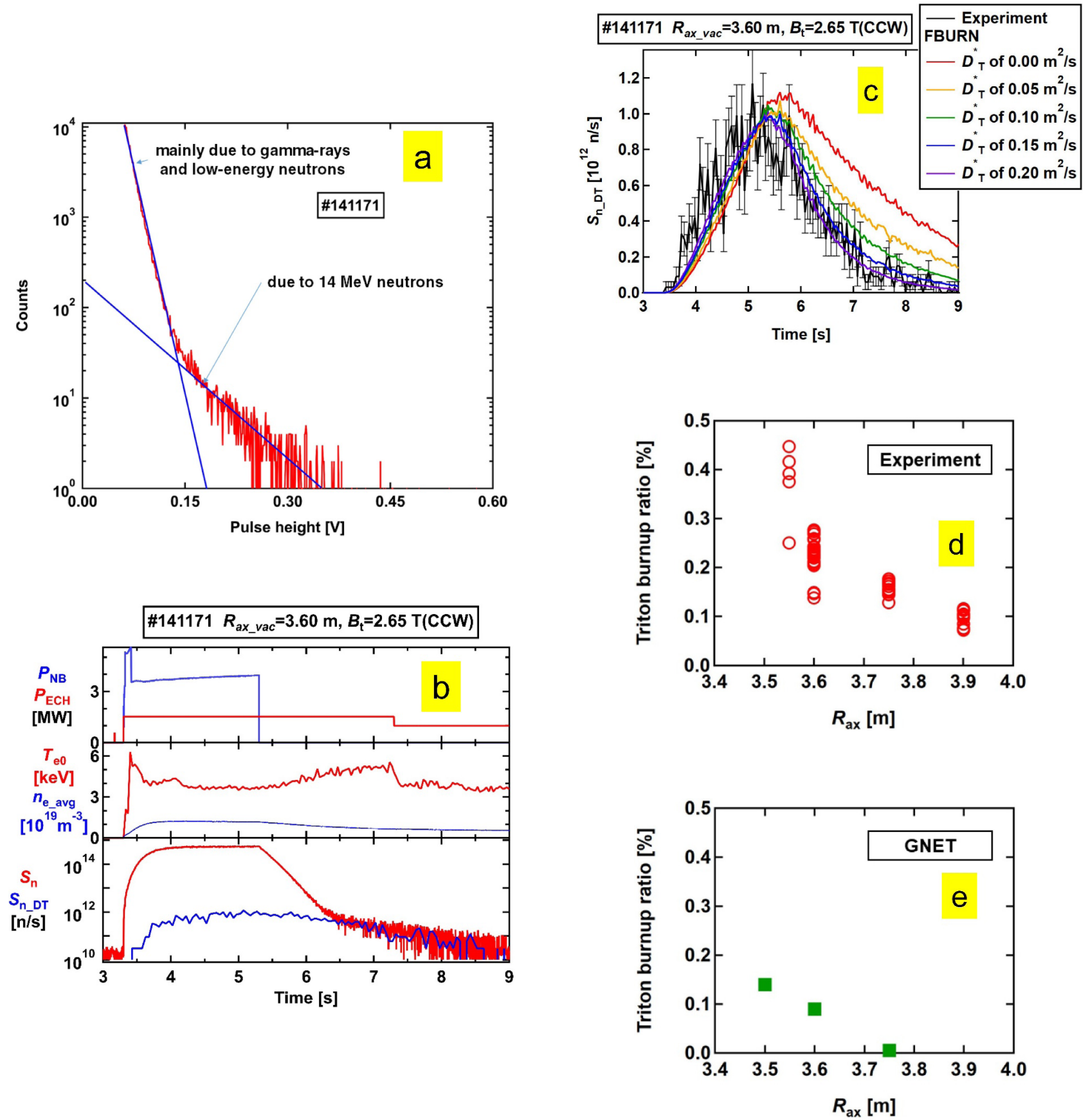

Fig. 15 (a) Pulse height spectra of NIFS Sci-Fi signal. There are low-height and high-height components. Reproduced with permission from K. Ogawa et al., Nucl. Fusion 58, 034002 (2018) [98]. (b) Time evolution of triton burnup experiment. Reproduced with permission from K. Ogawa et al., Nucl. Fusion 58, 034002 (2018) [98]. Here, $S_{\mathrm{n}_{-} \text {DT }}$ represents the total DT neutron emission rate. (c) Comparison of total DT neutron emission rate measured during the experiment and calculated by utilizing the FBURN code. Reproduced with permission from K. Ogawa et al., Plasma Phys. Control. Fusion 60, 9 (2018) [57]. Here, $D_{\mathrm{T}}^{*}$ indicates the radial diffusion coefficient of tritons. Magnetic axis dependence of triton burnup ratio obtained from the experiment (d) and predicted by utilizing the GNET code (e). Reproduced with permission from K. Ogawa et al., Nucl. Fusion 59, 076017 (2019) [52].

When the time evolution of DT neutrons was compared with the results of the FBURN code, it is observed that the radial diffusion coefficient is $0.15-0.2 \mathrm{~m}^{2} / \mathrm{s}$. The dependence of the triton burnup ratio on the magnetic axis position was evaluated (Fig. 15 (d)). Herein, the triton burnup ratio is defined as the shot-integrated DT neutron yield divided by the shot-integrated DD neutron yield. Also, the shot-integrated DT neutron yield and the shot-integrated DD neutron yield were measured using the Sci-Fi detector calibrated by the NAS and the NFM, respectively. It has been reported that the triton burnup ratio substantially increases with the inward shift of the magnetic axis [52], as predicted by employing the GNET code [106]. The improvement of the triton burnup may be due to the improve- 
ment of helically trapped beam ion confinement. Furthermore, the maximum triton burnup ratio was achieved to be $0.45 \%$ at a magnetic axis position of $3.55 \mathrm{~m}$. The obtained maximum triton burnup ratio equals the triton burnup ratio reported in the ASDEX-Upgrade and $\operatorname{KSTAR}[107,108]$, whose minor radii of the plasma are almost the same as that of LHD. It is shown that LHD has an equivalent confinement ability of alpha particles to that in tokamaks. Using a collisionless Lorentz orbit (LORBIT) code [109], an evaluation of the first orbit loss was performed to understand the magnetic configuration effect of the triton burnup ratio [110]. It was observed that the confinement of tritons having helically-trapped and transition orbits improved with the inward shift of the magnetic axis. Additionally, the loss points of triton measured by applying a tritium imaging plate technique were compared with the first orbit loss results [111]. The LORBIT code demonstrated that the loss point of triton changes based on the direction of the toroidal magnetic field. The asymmetry of the amount of tritium remaining on the right and left sides of the divertor plate agrees with the frequency of the experiments. In the first $\mathrm{D}$ experimental campaign, the ratio of counterclockwise toroidal magnetic field direction day to clockwise toroidal magnetic field direction days was approximately $5: 1$.

\subsection{NF detector}

An NF detector was installed on the lower port of LHD and used for measuring the rapid change of neutron flux due to MHD instabilities (Fig. 16 (a)) [112]. An EJ410 scintillator, whose diameter and thickness are 1-inch and 0.75 -inch, respectively, coupled with a 1 -inch photomultiplier tube (R9800, Hamamatsu Photonics KK), was chosen as the detector. Moreover, the anode signal is fed into the low-impedance preamplifier (C7319, Hamamatsu Photonics K. K.) and obtained using the data acquisition system (PXI-6133, National Instruments) with $1 \mathrm{MHz}$ sampling. In order to be placed near LHD, where the magnetic field strength is relatively high, a relatively long iron and Permalloy C magnetic shield having a length of $300 \mathrm{~mm}$ was utilized for the R9800 photomultiplier tube. Note that although the typical magnetic field strength around the NF detector position is approximately $40 \mathrm{mT}$, the typical magnetic field strength inside the magnetic shield is $0.01 \mathrm{mT}$.

Measurement of the neutron flux using the NF detector was performed in relatively high $S_{\mathrm{n}}$ discharges. The comparison of an NF detector signal with $S_{\mathrm{n}}$ measured by applying the NFM showed that the NF detector signal linearly increases with $S_{\mathrm{n}}$ at least to $2 \times 10^{15} \mathrm{n} / \mathrm{s}$. The conversion factor of the NF detector signal to $S_{\mathrm{n}}$ in this discharge was observed to be $2.9 \times 10^{14} \mathrm{n} / \mathrm{s} / \mathrm{V}$. Furthermore, the measurement of the neutron flux in an EIC discharge was performed using the NF detector. Note that the fluctuations in the signal of the NF detector were because of MHD instabilities excited by bulk plasma pressure. Moreover, $S_{\mathrm{n}}$
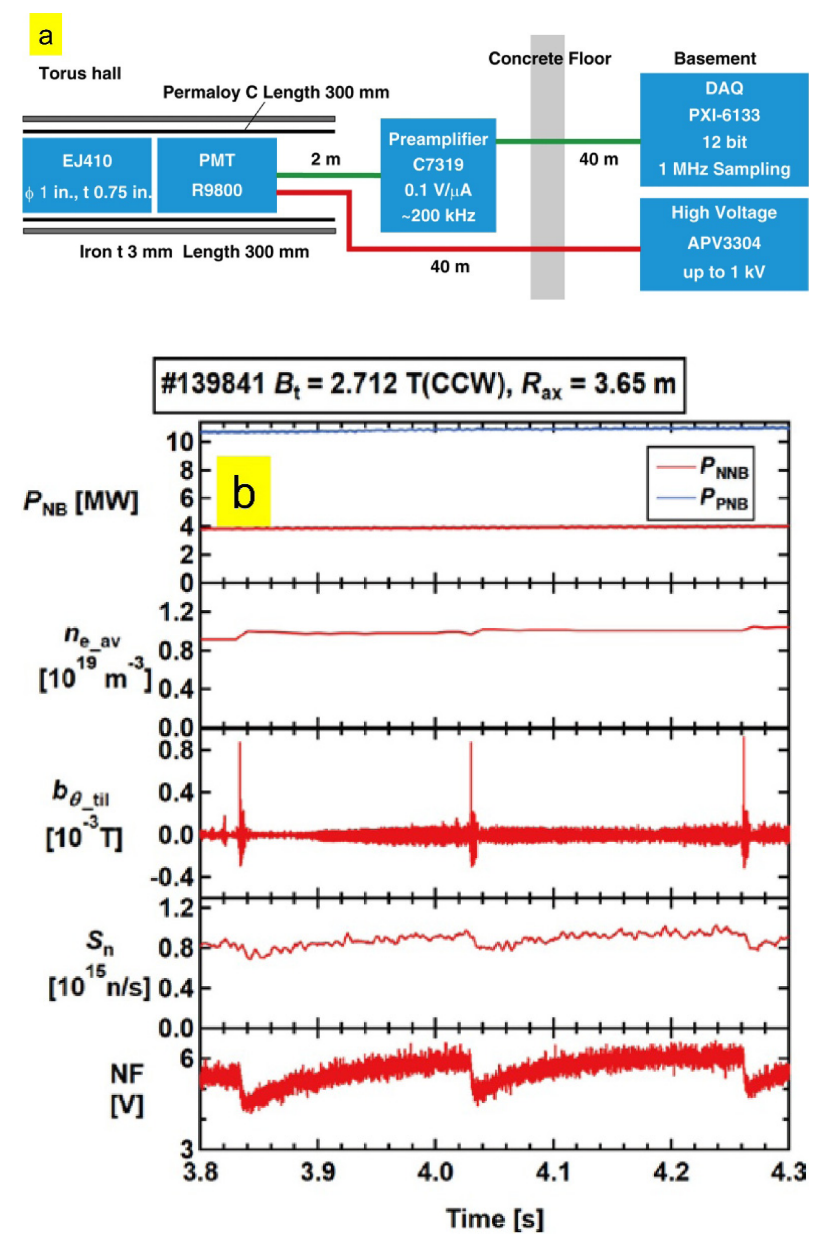

Fig. 16 (a) Block diagram of the NF detector. (b) The total neutron emission rate with a fast time response was measured using the NF detector. Reproduced with permission from K. Ogawa et al., Plasma Fusion Res. 13, 3402068 (2018) [112]. Here, $b_{\theta_{-} \text {til }}$, and NF represent the magnetic fluctuation amplitude measured by the magnetic probe, and the NF detector signal, respectively.

measured using the NFM and the neutron flux measured using the NF detector decreased by approximately $20 \%$ in synchronization with EIC bursts (Fig. 16 (b)). The drop of $S_{\mathrm{n}}$ within $2 \mathrm{~ms}$ was clearly observed using the NF detector.

\section{Summary}

Neutron diagnostics developed for LHD deuterium operation were installed and are operating properly from FY2017 to FY2019. Based on the neutron diagnostic plan in LHD, the NFM, NAS, VNC, Sci-Fi detector, and NF were established. NFM having a wide dynamic range, fast time response, and high noise tolerance was utilized for measuring $S_{\mathrm{n}}$. The density and magnetic configuration dependence of $S_{\mathrm{n}}$ of LHD plasmas, fuel ion temperature evaluation, global confinement of beam ions, investigation of knock-on tail formation, and study of the beam-beam nonlinear collision were conducted. To measure shotintegrated DD or DT neutron fluence, a NAS having two 
irradiation ends was utilized. Additionally, a cross-check of the NFM, anisotropy of DD neutron emission, and neutron spectra measurements were performed. To measure the radial profile of the neutron emission, VNCs installed at three toroidal angles were utilized. Measurement of the neutron emission profile in N-NB or P-NB-heated plasmas and the study of energetic ion transport owing to energetic ion driven MHD instability were conducted. Three types of Sci-Fi detectors were developed for time-resolved measurement of the DT neutron flux. The time evolution of the secondary DT neutron emission rate was compared with the numerical simulation based on the classical confinement of energetic ions. Furthermore, the dependence of DT neutron emission rate on the magnetic axis position was surveyed. An NF detector having a fast time response was utilized to measure the rapid change of $S_{\mathrm{n}}$. In an EIC discharge, the rapid decay of $S_{\mathrm{n}}$ was measured. Energetic particle confinement studies in LHD were largely advanced by utilizing integrated neutron diagnostics from FY2017 to FY2019.

[1] A. Fasoli et al., Nucl. Fusion 47, S264 (2007).

[2] W.W. Heidbrink and G.J. Sadlers, Nucl. Fusion 34, 535 (1994).

[3] O. Kaneko et al., Nucl. Fusion 53, 104015 (2013).

[4] Y. Takeiri et al., Nucl. Fusion 57, 102023 (2017).

[5] M. Isobe et al., Fusion Sci. Technol. 58, 426 (2010).

[6] M. Osakabe et al., Rev. Sci. Instrum. 75, 3601 (2004).

[7] M. Osakabe et al., Nucl. Fusion 46, S911 (2006).

[8] M. Osakabe et al., Fusion Sci. Technol. 58, 131 (2010).

[9] M. Osakabe et al., Rev. Sci. Instrum. 72, 788 (2001).

[10] M. Isobe et al., Rev. Sci. Instrum. 41, 1273 (2001).

[11] E.A. Veshchev et al., Rev. Sci. Instrum. 77, 10F129 (2006).

[12] S. Kamio et al., Nucl. Fusion 60, 112002 (2020).

[13] H. Wang et al., Phys. Plasmas 22, 092507 (2015).

[14] M. Nishiura et al., Rev. Sci. Instrum. 75, 3646 (2004).

[15] K. Ogawa et al., Plasma Fusion Res. 3, S1082 (2008).

[16] K. Ogawa et al., J. Plasma Fusion Res. SERIES 8, 655 (2009).

[17] K. Ogawa et al., Plasma Fusion Res. 4, 033 (2009).

[18] K. Ogawa et al., Nucl. Fusion 50, 084005 (2010).

[19] K. Ogawa et al., Plasma Fusion Res. 7, 2402014 (2012).

[20] K. Ogawa et al., Nucl. Fusion 52, 094013 (2012).

[21] K. Ogawa et al., Nucl. Fusion 53, 053012 (2013).

[22] K. Ogawa et al., Plasma Fusion Res. 9, 340297 (2014).

[23] K. Ogawa et al., Plasma Phys. Control. Fusion 56, 094005 (2014).

[24] R. Seki et al., Nucl. Fusion 59, 096018 (2019).

[25] R. Seki et al., J. Plasma Phys. 86, 815860502 (2020).

[26] Y. Takeiri, IEEE Trans. Plasma Sci. 46, 2348 (2018).

[27] Y. Takeiri et al., IEEE Trans. Plasma Sci. 46, 1141 (2018).

[28] M. Osakabe et al., Fusion Sci. Technol. 72, 199 (2018).

[29] J. Fujita, Fusion Eng. Des. 34-35, 11 (1997).

[30] M. Sasao et al., Fusion Eng. Des. 34-35, 595 (1997).

[31] M. Isobe et al., Rev. Sci. Instrum. 81, 10D310 (2010).

[32] N. Javis, Plasma Phys. Control. Fusion 36, 209 (1994).

[33] T. Nishitani et al., Fusion Eng. Des. 136, Part A, 210 (2018).

[34] H. Kawase et al., IEEE Trans. Plasma Sci. 47, 462 (2019).
[35] N. Nishio et al., Rev. Sci. Instrum. 81, 10 D306 (2010).

[36] N. Nishio et al., Plasma Fusion Res. 6, 2405115 (2011).

[37] Y. Nakano et al., Rev. Sci. Instrum. 85, 11E116 (2014).

[38] Y. Nakano et al., Plasma Fusion Res. 9, 3405141 (2014).

[39] T. Nishitani et al., Plasma Fusion Res. 11, 2045057 (2016).

[40] T. Nishitani et al., Fusion Eng. Des. 123, 1020 (2017).

[41] K. Ikeda et al., Nucl. Fusion 59, 076009 (2019).

[42] H. Nakanishi et al., Fusion Eng. Des. 129, 259 (2018).

[43] M. Isobe et al., Rev. Sci. Instrum. 85, $11 \mathrm{E} 114$ (2014).

[44] D. Ito et al., Plasma Fusion Res. 16, 1405018 (2021).

[45] M. Isobe et al., IEEE Trans. Plasma Sci. 46, 2050 (2018).

[46] C.Z. Chen and M.S. Chance, Phys. Fluids 29, 3695 (1986).

[47] L. Chen, Phys. Plasmas 1, 1519 (1994).

[48] K. Ogawa et al., Plasma Sci. Technol. 19, 025601 (2017).

[49] K. Ogawa et al., Nucl. Fusion 57, 086012 (2017).

[50] M. Isobe et al., Nucl. Fusion 58, 082004 (2018).

[51] K. Ogawa et al., Plasma Fusion Res. 12, 1202036 (2017).

[52] K. Ogawa et al., Nucl. Fusion 59, 076017 (2019).

[53] S. Murakami et al., Trans. Fusion Technol. 27, 256 (1995).

[54] P. Vincenzi et al., Plasma Phys. Control. Fusion 58, 124008 (2016).

[55] R. Seki et al., Plasma Fusion Res. 14, 3402126 (2019).

[56] K. Ogawa et al., Plasma Fusion Res. 14, 1202159 (2019).

[57] K. Ogawa et al., Plasma Phys. Control. Fusion 60, 9 (2018).

[58] T. Nishitani et al., Plasma Fusion Res. 13, 3402024 (2018).

[59] H. Nuga et al., Plasma Fusion Res. 14, 3402075 (2019).

[60] H. Nuga et al., J. Plasma Phys. 86, 815860306 (2020).

[61] S. Murakami et al., Nucl. Fusion 46, L19 (2002).

[62] S. Murakami et al., Nucl. Fusion 46, S425 (2006).

[63] H. Nuga et al., Nucl. Fusion 59, 016007 (2019).

[64] H. Matsuura et al., Nucl. Fusion 60, 066007 (2020).

[65] M.G. McCoy, A.A. Mirin and J. Killeen, Comput. Phys. Commun. 24, 37 (1981).

[66] R.W. Harvey et al., Nucl. Fusion 59, 106046 (2019).

[67] M. Kobayashi et al., Nucl. Fusion 59, 126003 (2019).

[68] T. Tanaka et al., Plasma Fusion Res. 14, 3405162 (2019).

[69] N. Pu et al., Rev. Sci. Instrum. 88, 113302 (2017).

[70] M.I. Kobayashi et al., Plasma Fusion Res. 15, 2405043 (2020).

[71] T. Nishitani et al., IEEE Trans. Plasma Sci. 47, 12 (2019).

[72] D.A. Spong, Phys. Plasmas 18, 056109 (2011).

[73] T. Goorley et al., Initial MCNP6 release overview, Nucl. Technol. 180, (December), 298 (2012).

[74] S. Sugiyama et al., Plasma Fusion Res. 14, 3403123 (2019).

[75] S. Sugiyama et al., Nucl. Fusion 60, 076017 (2020).

[76] T. Tanaka et al., Fusion Eng. Des. 146, 496 (2019).

[77] P.J. Griffin et al., User's Manual for SNL-SAND-II Code, Sandia National Labs, 1994 Report SAND-93-3957.

[78] K. Miyamoto, Plasma Physics for Controlled Fusion (Berlin: Springer, 2016) p.410.

[79] K. Ogawa et al., Rev. Sci. Instrum. 89, 113509 (2018).

[80] S. Sangaroon et al., Rev. Sci. Instrum. 91, 083505 (2020).

[81] K. Ogawa et al., Rev. Sci. Instrum. 85, 11E110 (2014).

[82] Y. Uchida et al., Rev. Sci. Instrum. 85, 11E118 (2014).

[83] Y. Uchida et al., Rev. Sci. Instrum. 88, 083504 (2017).

[84] R. Seki et al., Plasma Fusion Res. 10, 1402077 (2015).

[85] S. Kamio et al., J. Instrum. 14, C08002 (2019).

[86] H. Kawase et al., Plasma Fusion Res. 13, 3402122 (2018). 
[87] K. Ogawa et al., Plasma Phys. Control. Fusion 60, 044005 (2018).

[88] X.D. Du et al., Phys. Rev. Lett. 114, 155003 (2015).

[89] X.D. Du et al., Nucl. Fusion 56, 016002 (2016).

[90] X.D. Du et al., Phys. Rev. Lett. 118, 125001 (2017).

[91] H. Takahashi et al., Nucl. Fusion 58, 106028 (2018).

[92] T. Bando et al., Nucl. Fusion 58, 082025 (2018).

[93] K. Ogawa et al., Nucl. Fusion 58, 044001 (2018).

[94] K. Ogawa et al., Nucl. Fusion 60, 112011 (2020).

[95] N. Pu et al., Rev. Sci. Instrum. 89, $10 \mathrm{I} 105$ (2018).

[96] N. Pu et al., J. Instrum. 14, P10015 (2019).

[97] N. Pu et al., Nucl. Instrum. Methods Phys. Res. A 969, 164000 (2020)

[98] K. Ogawa et al., Nucl. Fusion 58, 034002 (2018).

[99] E. Takada et al., Plasma Fusion Res. 11, 2405020 (2016).

[100] E. Takada et al., Rev. Sci. Instrum. 90, 043503 (2019).

[101] K. Ogawa et al., Rev. Sci. Instrum. 89, $10 \mathrm{I101}$ (2018).
[102] J. Jo et al., Rev. Sci. Instrum. 89, 101118 (2018).

[103] J.P. Koschinsky et al., Contrib. Plasma Phys. 60, e201900186 (2020).

[104] K. Ogawa et al., Nucl. Fusion 58, 034002 (2018).

[105] N. Pu et al., Plasma Fusion Res. 13, 3402121 (2018).

[106] M. Homma et al., Plasma Fusion Res. 10, 3403050 (2015).

[107] M. Hoek, H.S. Bosch and W. Ullrich, "Triton burnup measurement at ASDEX Upgrade by neutron foil activation" IPP-Report IPP-1/320 (1999).

[108] J. Jo et al., Rev. Sci. Instrum. 87, 11D828 (2016).

[109] M. Isobe et al., J. Plasma Fusion Res. SERIES 8, 330 (2009).

[110] K. Ogawa et al., Plasma Sci. Technol. 21, 025102 (2019).

[111] S. Masuzaki et al., Phys. Scripta T171, 014068 (2020).

[112] K. Ogawa et al., Plasma Fusion Res. 13, 3402068 (2018). 